3 Research Square

\title{
4R Tau Modulates Cocaine-Associated Memory Through Adult Hippocampal Neurogenesis
}

\section{Hongchun Li}

Sichuan University West China Hospital

Wei Xu

Sichuan University West China Hospital

\section{Denian Wang}

Sichuan University West China Hospital

\section{Qiyao Fang}

Sichuan University West China Hospital

\section{Liang Wang}

Sichuan University West China Hospital

\section{Xuemei Wan}

Sichuan University West China Hospital

Jiamei Zhang

Sichuan University West China Hospital

Yiming Hu

Sichuan University West China Hospital

Huifang Li

Sichuan University West China Hospital

Jie Zhang

Sichuan University West China Hospital

Zhen Yang

Sichuan University West China Hospital

Chunqi Liu

Sichuan University West China Hospital

Xiaocong Liu

Sichuan University West China Hospital

Yonghai Wang

Yantai University

Bin Liu

Sichuan University West China Hospital

\section{Ying Zhao}

Sichuan University West China Hospital

Qian Bu 
Sichuan University West China Hospital

\section{Hongbo Wang}

Yantai University

Jingwei Tian

Yantai University

\section{Yinglan Zhao}

Sichuan University West China Hospital

Xiaobo Cen ( $\nabla$ xbcen@scu.edu.cn )

Sichuan University West China Hospital

\section{Research article}

Keywords: 4R Tau, PI3K-p85, cocaine associated-memory, adult hippocampal neurogenesis, PI3K-AKT signaling, LY294002

Posted Date: September 25th, 2020

DOl: https://doi.org/10.21203/rs.3.rs-74944/v1

License: (c) (7) This work is licensed under a Creative Commons Attribution 4.0 International License. Read Full License 


\section{Abstract}

Background: Drug memory that generally develops with drug-paired contextual stimuli and drug administration is critical for the development, persistence and relapse of drug addiction. Previous studies have suggested that adult hippocampal neurogenesis (AHN) plays a role in cocaine memory formation; however, the underlying mechanism is not fully understood.

Methods: Conditioned place preference (CPP), self-administration and locomotor activity were used to investigate the role of Tau in cocaine-associated memory formation. Virus-mediated gene transfer, western blot, immunohistochemistry, flow cytometry analysis, Tau-interacting proteomics, coimmunoprecipitation, and mutation of $4 \mathrm{R}$ Tau were performed.

Results: Hippocampal expression of Tau was significantly decreased during the cocaine-associated memory formation. Genetic overexpression of four microtubule-binding repeats Tau (4R Tau) in the hippocampus disrupted cocaine memory by suppressing AHN. Furthermore, 4R Tau directly interacted with phosphoinositide 3-kinase (PI3K)-p85 and impaired its nuclear translocation and PI3K-AKT signaling, processes required for hippocampal neuron proliferation.

Conclusions: 4R Tau modulates cocaine memory formation by disrupting AHN, suggesting a novel mechanism underlying cocaine memory formation and provide a new strategy for the treatment of cocaine addiction.

\section{Background}

Drug addiction is characterized by compulsive drug seeking behaviors, repeated attempts at abstinence and high rates of relapse. Similar to the other memories, long-lasting drug-associated memory formation is closely associated with the rewarding effect of drugs and environmental cues related to drug exposure. Re-exposure to drug-associated contextual cues reactivates drug memory and promotes compulsive drugseeking behaviors during the period of abstinence [1]. An understanding of the neurobiological mechanisms underlying drug memory formation is important for exploring effective strategies for drug addiction treatment and relapse prevention.

The hippocampus plays a critical role in the formation of drug-associated contextual memory [2-5]. Accumulating evidence has suggested that hippocampus-dependent activities, such as memory encoding and mood regulation, are regulated by adult hippocampal neurogenesis (AHN), a unique phenomenon that confers neuroplasticity to the hippocampal circuitry and plays a significant role in neurodegenerative and psychiatric disorders [6-8]. In this process, new neurons undergo continual proliferation, migration, differentiation and integration into the existing neuronal circuitry of the dentate gyrus (DG), eventually modulating the formation and updating of hippocampus-dependent memory $[9,10]$. AHN has been recently considered an important factor in the establishment and maintenance of addiction memory [1113]. When animals are subjected to pro-AHN manipulations, such as exercise, the subsequently acquired 
cocaine memory becomes stronger and more resistant to extinction [14]. However, the precise mechanism underlying the role of $\mathrm{AHN}$ in cocaine memories formation has not been completely elucidated.

Tau is a microtubule-associated scaffolding protein whose major function is the stabilization of microtubules and promotion of microtubule polymerization [15]. Tau isoforms containing either three or four microtubule-binding repeats (3R Tau or 4R Tau) exhibit approximately equal expression in the normal adult human brain; however, $4 \mathrm{R}$ Tau is the predominant isoform expressed in the adult murine brain. Tau was recently shown to be associated with the proliferation of newborn hippocampal granule neurons that require a high level of cytoskeletal plasticity to divide, proliferate and differentiate in response to external stimuli [16-18]. During early brain development, 3R Tau predominates in the neonatal brain and exhibits a lower affinity for microtubules than 4R Tau. Therefore, 3R Tau participates in the morphological differentiation and migration of immature neurons. In contrast, 4R Tau binds with higher affinity to microtubules, thereby maintaining the cytoskeletal stability and neuronal integrity [19, 20]. To date, the role of Tau isoforms in the formation of drug-associated memory is unknown. In the present study, our findings reveal a novel mechanism by which 4R Tau modulates cocaine memory formation by regulating $\mathrm{AHN}$.

\section{Materials And Methods}

\section{Animals}

Male C57BL/6J wild-type (WT) mice (8-12 weeks old) were purchased from Vital River Laboratory Animal Technology Co. Ltd. (Beijing, China). Tau ${ }^{-/-}$knockout (Tau-KO) mice on the C57BL/6J background were obtained from Jackson Laboratories (\#007251, USA). Male and female homozygous mice were bred to generate $\mathrm{Tau}^{-/-}$homozygous mice, and 8-12 week-old male homozygous Tau-KO mice and WT littermates (C57BL/6J) were used in this study. All mice were housed in the animal room on a standard 12-h light/12-h dark cycle with a constant temperature and food and water available ad libitum. All experimental procedures and use of the animals were conducted in accordance with the guidelines established by the Association for Assessment and Accreditation of Laboratory Animal Care and the Institutional Animal Care and Use Committee of Sichuan University. All efforts were made to minimize the suffering of the mice.

\section{Behavioral paradigm}

Mice were acclimated for one week before experiments and habituated to handling for $2 \mathrm{~d}$ before each behavioral test. Conditioned place preference (CPP) and self-administration were used to assess cocainecue memory formation. The home cage CPP, food CPP and locomotor activity were employed as control behavioral tests. All behavioral experiments were performed in a double-blind manner.

\section{Conditioned place preference (CPP)}


The CPP test was conducted using a standard three-chambered apparatus equipped with two large conditioning compartments (black and white) that differed in their flooring (bar and grid) and a small middle chamber (grey, smooth PVC floor) that connected the two large compartments. Prior to each session, animals were habituated to the chambers for at least 10 min for a continuous $2 \mathrm{~d}$.

\section{Pre-test session}

Baseline preference was assessed by placing the mice in the middle chamber and allowing them to habituate the entire chambers freely for $15 \mathrm{~min}$; an initial measurement of baseline preference was defined as the time spent in the black chamber subtracted from the time spent in the white chamber $\left(T_{i m e}{ }_{\text {pre-test }}\right)$. Animals were excluded from the test if they showed a strong unconditioned preference for either side chamber (chamber bias $>300 \mathrm{~s}$ ).

\section{CPP training}

Animals were randomly assigned to two groups and trained for $6 \mathrm{~d}$ with alternating injections of cocaine ( $20 \mathrm{mg} / \mathrm{kg}$ or $2.5 \mathrm{mg} / \mathrm{kg}$, i.p) or saline injections in both compartments. They were confined to the conditioning or unconditioning chambers for $30 \mathrm{~min}$ after the injection and then returned to their home cages. On the test day, the animals were placed in the middle compartment and the time of spent in the two compartments was recorded for $15 \mathrm{~min}$. We also defined as the time spent in the black chamber

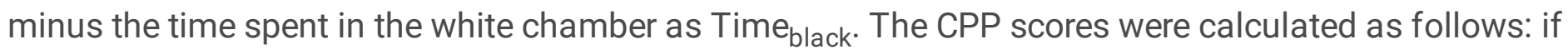
Time $_{\text {black }}$ was positive (the mouse preferred the black chamber), namely, Time black $_{\text {minus Time }}$ pre-test, positive scores indicated a reinforced preference, and negative scores indicated a reversed preference. If Time $_{\text {black }}$ was negative (the mouse preferred the white chamber), Time black minus Time pre-test $_{\text {, negative }}$ scores indicated a reversed preference and positive scores indicated a reinforced preference. Generally, cocaine strongly reverses preference and results in a positive CPP score, and the CPP score was defined as the extent of the shift in preference after the cocaine injection.

\section{Home cage CPP}

Mice were exposed to cocaine as described for consolidation training, and then they were not confined to the conditioning or unconditioning chambers, but were returned to their home cages without any context exposure to the test apparatus. On the test day, mice were placed in the middle connecting chamber, and the time spent in each chamber was calculated as described in previous experiments to evaluate the CPP score.

\section{Food CPP}

The food CPP test used a similar apparatus and methodology as described above. Mice were restricted from food for one week before the test, and their weight was maintained at $80 \%$ of their original body weight. During the food conditioning sessions, the food-induced CPP group was transferred to the foodpaired chamber (2-3 $\mathrm{g}$ of food were placed in the chamber) for $30 \mathrm{~min}$. In the non-food conditioning sessions, mice were assigned to the non-food-paired chamber for $30 \mathrm{~min}$. After conditioning training for $3 \mathrm{~h}$, animals were only fed once daily $(1 \mathrm{~h})$. The alternating sessions of conditioning were repeated 3 
times (a total of $6 \mathrm{~d}$ ). On the test day, mice were placed in the central chamber and allowed to freely explore both compartments for $15 \mathrm{~min}$; the time spent in each chamber was recorded to calculate the CPP score.

\section{CPP and extinction}

Mice were exposed to the CPP protocol, and the test day course was repeated each day until the preference of the cocaine group for the conditioned compartment had returned to the habituation baseline.

\section{Cocaine self-administration}

Mice were anesthetized with sodium pentobarbital $(60 \mathrm{mg} / \mathrm{kg})$ and implanted with a single sterilized silastic catheter ( 0.51 ID $\times 0.94 \mathrm{~mm}$ OD, BB518-20, Scientific Commodities) into the right jugular vein. The distal end of the catheter was threaded through the skin on the back of the mice and exited the skin via a stainless steel guide cannula (RWD Life Science). After surgery, catheters were flushed with $0.1 \mathrm{ml}$ of a saline solution containing penicillin $(160000 \mathrm{U} / \mathrm{ml})$ and heparin $(30 \mathrm{U} / \mathrm{ml})$ daily. One week after recovering from surgery, animals were trained to self-administer intravenous injections of cocaine $(0.75 \mathrm{mg} / \mathrm{kg} /$ infusion) or saline during $2 \mathrm{~h}$ sessions daily over $10 \mathrm{~d}$ on a fixed ratio 1 (FR1) schedule in an operant chamber. The response to the active poke produced a cocaine injection and was accompanied with a blue light stimulus for $20 \mathrm{~s}$ and an audible tone for a 5-s timeout period. At the same time, the inactive poke failed to inject the drug and produce conditioning stimuli.

\section{Locomotor activity}

The locomotor activity was measured as the distance traveled. Animals were acclimated to the chambers $(48 \mathrm{~cm} \times 48 \mathrm{~cm}$ ) equipped with a camera for 15 min once a day for a consecutive $2 \mathrm{~d}$. Before the test, the baseline locomotor activity was not statistically different between groups. In the following week, animals were injected cocaine (20 mg/kg, i.p) or an equal volume of saline, immediately placed in the locomotor activity chamber, and allowed to explore for $15 \mathrm{~min}$. The distance traveled was measured daily for one week, and automated tracking was performed with EthoVision 7.0 software (EthoVision 7.0; Noldus Information Technology, Leesburg, VA).

\section{Drugs}

Cocaine was purchased from the National Institute for the Control of Pharmaceutical and Biological Products (Beijing, China) and dissolved in saline. LY294002 (S1105, Selleck) were dissolved in saline containing 4\% DMSO, 30\% PEG300 and 5\% Tween 80.

\section{Stereotactic surgery and inhibitor administration}

Animals were anesthetized with sodium pentobarbital $(60 \mathrm{mg} / \mathrm{kg})$ and mounted in a standard stereotaxic instrument (RWD Life Science). After shaving the hair and cleaning the incision site with medical-grade alcohol, the scalp was incised to expose the skull and permanent bilateral guide cannulas (RWD Life Science) were bilaterally implanted into the dorsal dentate gyrus (dDG) (AP, - 2.0 mm; ML, $\pm 1.4 \mathrm{~mm}$; DV, 
- $2.2 \mathrm{~mm}$ ) with stereotaxic instruments. Dental cement was used to anchor the guide cannula and the stainless steel stylet blocker was inserted into each cannula to prevent blockage and infection. All mice were used for subsequent training after one week of recovery from surgery.

LY294002, a specific inhibitor of the phosphoinositide 3-kinase (PI3K)-AKT signaling pathway, was also dissolved in saline containing 4\% DMSO, 30\% PEG300 and 5\% Tween 80 at a final concentration of $4.6 \mu \mathrm{g} / \mu \mathrm{l}$. LY294002 were administered bilaterally $(1 \mu \mathrm{l} / \mathrm{side}, 0.5 \mu \mathrm{l} / \mathrm{min})$ with a micro-injector, $15 \mathrm{~min}$ before cocaine or saline administration during the cocaine-associated memory formation.

\section{Adeno-associated virus injection}

The adeno-associated viruses (AAVs) AAV2/8-CMV-2N4R-3Flag $\left(1.83 \times 10^{13} \mathrm{vg} / \mathrm{ml}\right)$, AAV2/8-CMV-2N4R2A-mNeonGreen-3Flag $\left(8.53 \times 10^{12} \mathrm{vg} / \mathrm{ml}\right)$ and AAV2/8-CMV-mutant 2N4R-2A-mNeonGreen-3Flag $(2.83 \times$ $10^{12} \mathrm{vg} / \mathrm{ml}$ ) were purchased from Obio Technology Co. Ltd. (Shanghai, China). Point mutations of arginine 205, proline 208 and proline 210 to alanine were synthesized in the murine full-length Tau amino acid sequence, as these three residues are normally important for the interaction of PI3K-p85 SH3 domains, and their mutation has previously been shown to attenuate PI3K-p85 binding to human 2N4R Tau [21]. The AAV2/8-CMV-2N4R-3Flag, AAV2/8-CMV-2N4R-2A-mNeonGreen-3Flag and AAV2/8-CMVmutant 2N4R-2A-mNeonGreen-3Flag were constructed for use in animal studies.

The coding regions of 2N4R Tau were amplified from cDNAs obtained from C57BL/6J mice by PCR and cloned into pAAV-CMV-MCS-3Flag and pAAV-CMV-MCS-2A-mNeongreen-3Flag to produce AAV-2N4R Tau or mutant 2N4R Tau, respectively. The vector was confirmed by sequencing. The recombinant plasmids were then packaged into AAV2/8 particles together with the AAV helper plasmid and purified using an iodixanol step-gradient ultracentrifugation method. Recombinant AAVs were produced by transient transfection in HEK293T cells and the expression of 4R Tau was analyzed using western blotting.

Mice (8-12 weeks old) were anesthetized with sodium pentobarbital $(60 \mathrm{mg} / \mathrm{kg})$ and placed on a stereotaxic apparatus (RWD Life Science) to inject the virus into the dDG (AP, $-2.0 \mathrm{~mm} ; \mathrm{ML}, \pm 1.4 \mathrm{~mm} ; \mathrm{DV}$, $-2.2 \mathrm{~mm}$ ). After shaving the hair and cleaning the incision site with medical-grade alcohol, the scalp was incised to expose the skull and the connective tissue was gently removed from the skull surface with cotton swabs. Small craniotomy holes were drilled with a skull rotor (RWD Life Science) for virus injection. The micro syringe needles were used to bilaterally infuse the tissue with AAV2/8-CMV-2N4R3Flag (0.2 $\mu \mathrm{l}, 0.05 \mu \mathrm{l} / \mathrm{min})$, AAV2/8-CMV-2N4R-2A-mNeonGreen-3Flag $(0.4 \mu \mathrm{l}, 0.05 \mu \mathrm{l} / \mathrm{min})$ or AAV2/8CMV-mutant 2N4R-2A-mNeonGreen-3Flag $(1.2 \mu \mathrm{l}, 0.05 \mu \mathrm{l} / \mathrm{min})$. After each injection, the syringe was left in place for an additional 5 min and then slowly withdrawn to allow the virus to diffuse. Mice recovered for at least 3 weeks before behavioral tests.

\section{Lentiviral vector construction}

The lentiviral vectors (LV) pLenti-EF1a-CMV-2N4R-3Flag (3.39 × $\left.10^{8} \mathrm{TU} / \mathrm{ml}\right)$, pLenti-EF1a-CMV-mutant 2N4R-3Flag (3.41 × 108 TU/ml), pLenti-CMV-p85a-MYC-2A-mCherry-PGK-blasticidin $\left(3.94 \times 10^{8} \mathrm{TU} / \mathrm{ml}\right)$ 
and pLKD-CMV-EGFP-2A-Puro-U6-shRNA Tau $\left(5.44 \times 10^{8} \mathrm{TU} / \mathrm{ml}\right)$ were also purchased from Obio Technology Co. Ltd. (Shanghai, China). The pLenti-EF1a-CMV-2N4R-3Flag, pLenti-EF1a-CMV-mutant 2N4R-3Flag, pLenti-CMV-p85a-MYC-2A-mCherry-PGK-blasticidin and pLKD-CMV-EGFP-2A-Puro-U6-shRNA Tau were constructed for cell-based studies.

The coding regions of 2N4R Tau and PI3K-P85a were amplified from murine cDNAs using PCR and cloned into pLenti-EF1a-EGFP-P2A-Puro-CMV-MCS-3Flag and pLenti-CMV-MCS-MYC-2A-mCherry-PGKblasticidin vectors to produce LV-2N4R Tau, LV-mutant 2N4R Tau and PI3K-P85a, respectively. The Tau shRNA was cloned into pLKD-CMV-EGFP-2A-Puro-U6-shRNA and confirmed by sequencing. The sequences of the scrambled control shRNA and Tau shRNA were 5'-TTCTCCGAACGTGTCACGT-3' and 5'GGAGTTTGACACAATGGAA-3', respectively. Recombinant lentiviruses were also produced by transient transfection in the HEK293T cells and then the levels of 4R Tau and PI3K-p85 were analyzed using western blotting.

\section{Tissue isolation}

Mice were sacrificed by rapid decapitation at the end of the behavioral tests. The hippocampus or dentate gyrus (separated from the hippocampus) were removed from the brain, snap frozen in dry ice, and stored at $-80^{\circ} \mathrm{C}$ until the assay.

\section{Western blot}

Brain tissues and cells were lysed and proteins were extracted using a mammalian cell and tissue extraction kit (K269-500, Biovision) containing phosphatase inhibitors (4906845001, Roche) according to the manufacturer's protocols. The total protein concentration was analyzed with a Bradford assay kit (P0006, Beyotime). Twenty micrograms protein were loaded and separated on 10 or $12.5 \%$ sodium dodecyl sulfate-polyacrylamide gels. After separation, the gels were then transferred to a polyvinylidene difluoride (PVDF) membrane (IPVH00010, Millipore) in a mixed solution of Tris-glycine buffer and $20 \%$ $(\mathrm{v} / \mathrm{v})$ methanol. The membrane was blocked in TBST buffer containing 5\% non-fat dry milk (9999, Cell Signaling Technology) for $1 \mathrm{~h}$ at room temperature, and then incubated and gently shaken overnight with the primary antibody at $4{ }^{\circ} \mathrm{C}$. On the next day, after three washes with TBST for $15 \mathrm{~min}$, the blots were incubated with the secondary antibody at room temperature for $2 \mathrm{~h}$. Immunoreactivity was visualized using a chemiluminescence substrate (WBKLS0500, Millipore) with a chemiluminescence imagine system (CLINX, Shanghai, China). The optical density of each band was quantified using Chemi Analysis software (CLINX, Shanghai, China). The following antibodies were used for western blotting: mouse antiTau (1:1000, Thermo Scientific), mouse anti-3R Tau (1:1000, Millipore), mouse anti-4R Tau (1:1000, Millipore), rabbit anti-CAMP response element-binding protein (CREB; 1:1000, Cell Signaling Technology), rabbit anti-p-CREB (S 133) (1:1000, Cell Signaling Technology), rabbit anti-extracellular signal-regulated kinase (ERK)1/2 (1:1000, Cell Signaling Technology), rabbit anti-p-ERK1/2 (T 202/T 204) (1:1000, Cell Signaling Technology), rabbit anti-cofilin (1:1000, Abcam), rabbit anti-p-cofilin (S 3) (1:1000, Cell Signaling Technology), rabbit anti-c-fos (1:1000, Cell Signaling Technology), mouse anti-Flag (1:5000, Sigma), mouse anti-2A (1:1000, Millipore), rabbit anti-PI3K-p85 (1:1000, Cell Signaling Technology), rabbit 
anti-PI3K-p110a (1:1000, Abcam), rabbit anti-AKT (1:1000, Abcam), rabbit anti-p-AKT (S 473) (1:1000, Cell

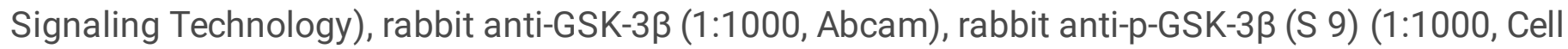
Signaling Technology), rabbit anti- $\beta$-catenin (1:1000, Abcam), rabbit anti-p- $\beta$-catenin (S 33/37/T 41) (1:1000, Cell Signaling Technology), rabbit anti- $\beta$-actin (1:1000, Cell Signaling Technology), mouse antiGAPDH (1:2000, ZSGB-BIO), rabbit anti-lamin B1 (1:1000, Abcam), goat anti-mouse (HRP) (SAB), goat anti-rabbit (HRP) (SAB), goat anti-mouse light chain-specific (HRP), and mouse anti-rabbit light chainspecific (HRP) (Jackson ImmunoResearch).

\section{Immunohistochemistry}

Animals that had undergone behavioral training were deeply anesthetized with sodium pentobarbital $(60 \mathrm{mg} / \mathrm{kg}$ ) and perfused transcardially with phosphate-buffered saline (PBS), followed by ice-cold $4 \%$ paraformaldehyde in 0.1 M PBS, pH 7.4. Brains were carefully extracted from the skull, postfixed with $4 \%$ PFA overnight, and then dehydrated with $30 \%$ sucrose at $4{ }^{\circ} \mathrm{C}$. Brains were sectioned into $45-\mu \mathrm{m}$ coronal slices using a freezing microtome (Leica, Germany) and stored in 12-well plates filled with a cryoprotectant solution (glycerol, ethylene glycol and $0.1 \mathrm{M}$ phosphate buffer, $\mathrm{pH} 7.4,1: 1: 2$ by volume) at $-20^{\circ} \mathrm{C}$ until processing for immunohistochemical staining.

The sections were washed with TBS 3 times (10 min each) and incubated with a blocking solution containing $0.3 \%$ TritonX-100 and 5\% normal donkey serum (017-000-121, Jackson ImmunoResearch) in TBS for $2 \mathrm{~h}$ at room temperature. Sections were then incubated with primary antibodies in the blocking solution at $4{ }^{\circ} \mathrm{C}$ overnight. On the next day, the sections were also washed with TBS- $0.3 \%$ TritonX-100 (TBST) 3 times, transferred to the blocking solution containing fluorescent dye-conjugated secondary antibodies, and then incubated for 1.5 hours at room temperature. After another three washes with TBST, sections were cover slipped with anti-fade mounting medium containing DAPI ( $\mathrm{H}-1200$, Vector), and confocal images were acquired with a laser confocal microscope (Nikon, Japan). The corresponding DG areas and cell numbers were determined using Image $J$ software (US National Institutes of Health, Bethesda, MD, USA) to assess the cell densities. The following antibodies were used for immunostaining: rabbit anti-Tau (1:100, Protech), mouse anti-3R Tau (1:200, Millipore), mouse anti-4R Tau (1:50, Biolegend), rat anti-BrdU (1:100, Abcam), mouse anti-Ki67 (1:100, BD), goat anti-sex determining region Ybox 2 (SOX2; 1:100, RD), rabbit anti-glial fibrillary acidic protein (GFAP; 1:100, Abcam), mouse anti-c-fos (1:100, Abcam), rabbit anti-doublecortin (DCX; 1:100, Abcam), donkey anti-mouse (Alexa Fluor 488) (1:500, Invitrogen), donkey anti-rabbit (Alexa Fluor 488) (1:500, Invitrogen), donkey anti-goat (Alexa Fluor 488) (1:500, Invitrogen), donkey anti-rabbit (Alexa Fluor 568) (1:500, Invitrogen), and donkey anti-rat (Alexa Fluor 647) (1:500, Jackson ImmunoResearch).

For BrdU staining, DNA was denatured by incubating the section in $2 \mathrm{M}$ hydrochloric acid for $30 \mathrm{~min}$ at $37^{\circ} \mathrm{C}$ and then renatured with $0.05 \mathrm{M}$ boric acid (28341, Thermo Scientific) at room temperature for $30 \mathrm{~min}$, followed by TBS rinses for $10 \mathrm{~min}$. Immunohistochemistry was then performed.

The cannula placements were confirmed in $5-\mu \mathrm{m}$ thick coronal sections using hematoxylin eosin (HE) staining, and images were acquired using a light microscope. Mice with misplaced cannulas were 
excluded from the statistical analysis.

\section{Immunocytochemistry}

Cells cultured on glass coverslips coated with poly-D-lysine (P6407, Sigma) were washed 3 times with PBS and then fixed with 4\% PFA for 10 min. After 3 washes with PBS, cells were blocked with blocking buffer ( $5 \%$ normal donkey serum and $0.3 \%$ TritonX-100 in PBS) for $1 \mathrm{~h}$ and then incubated with primary antibodies overnight. On the next day, coverslips were washed 3 times with PBS and incubated with the fluorescent dye-conjugated secondary antibodies for $1 \mathrm{~h}$ at room temperature. Coverslips were then washed 3 times with PBS, stained with Hoechst (H1399, Life Technologies) for 5 min, and mounted with anti-fade mounting medium ( $\mathrm{H}-1000$, Vector). The following antibodies were used for immunostaining: rabbit anti-PI3K-p85a (1:400, Abcam), mouse anti-4R Tau (1:100, Biolegend), donkey anti-mouse (Alexa Fluor 488) (1:500, Invitrogen), and donkey anti-rabbit (Alexa Fluor 568) (1:500, Invitrogen).

\section{Golgi staining}

Golgi staining was performed using the FD Rapid Golgi Stain Kit (PK401, FD Neurotechnologies) to label neurons in the dDG. Briefly, animals were deeply anesthetized with sodium pentobarbital $(60 \mathrm{mg} / \mathrm{kg})$, and the brains were immediately removed and rinsed with MilliQ water. Afterwards, the retrieved brains were immersed in equal parts of Solutions $A$ and $B$ (containing potassium dichromate, mercuric chloride, and potassium chromate) and stored at room temperature for 2 weeks in the dark. Tissues were subsequently rinsed, placed in the cryoprotectant solution, and stored at room temperature for at least 72 hours in the dark before cutting. The brain slices were cut in the coronal plane at approximately a $100-\mathrm{mm}$ thickness with the freezing microtome (Leica, Germany). Tissue sections were placed on poly-D-lysine-coated slides and dried in the dark. After drying, sections were transferred to distilled water, subsequently stained with a developing solution, and then successively dehydrated with $50,75,95$, and $100 \%$ ethanol. Finally, the sections were defatted with a xylene substitute and cover slipped with DPX mounting medium (06522, Sigma).

Images were acquired from prepared slides using a confocal microscope (Olympus, Japan). Each neuron was scanned at high magnification (100X, oil immersion) to ensure that all parts of the dendrites were intact. For each group, a minimum 5 neurons per slice were examined. At least 60 neurons were selected in one group. 3D neuronal reconstruction was also performed using a confocal microscope. The total length and dendrite spine density were measured using ImageJ software (US National Institutes of Health, Bethesda, MD, USA).

\section{Co-immunoprecipitation analysis}

Cells and tissues were harvested for the co-immunoprecipitation (co-IP) analysis using the simplified and reliable Pierce ${ }^{\text {TM }}$ Crosslink Magnetic IP/co-IP Kit (88805, Thermo Scientific). Briefly, the Tau or PI3K-p85 primary antibody was bound to $50 \mu \mathrm{l}$ of Protein A/G magnetic beads (B23201, Bimake) for 15 min and washed three times. After the incubation, the protein supernatants extracted using the mammalian cell and tissue extraction kit were collected, and the protein concentration was determined using a Bradford 
assay kit. The supernatant from each sample was incubated with the antibody-crosslinked beads overnight at $4{ }^{\circ} \mathrm{C}$. On the next day, beads were washed two times with IP lysis/wash buffer and one time with ultrapure water. Elution buffer was used to elute the bound antigen, and neutralization buffer was used to neutralize the low $\mathrm{pH}$. The supernatants were collected for western blot analysis.

\section{Cell culture and transfection}

Mouse neuroblastoma 2a (N2a) cells were cultured in Dulbecco's Modified Eagle's Medium (DMEM, C1195500BT, Gibco) containing 10\% fetal bovine serum (10099-141, Gibco) and grown in a 5\% $\mathrm{CO}_{2}$ atmosphere at $37^{\circ} \mathrm{C}$.

N2a cells were grown in 6-well plates to generate 2N4R Tau overexpression and Tau knockdown-resistant cells. DMEM was replaced with serum-free DMEM and incubated for $2 \mathrm{~h}$ before transfection, follow by incubations with the lentiviruses contain the mouse 2N4R Tau, mutant 2N4R Tau, PI3K-p85, Tau-shRNA (5'- GGAGTTTGACACAATGGAA-3') and the non-specific target oligonucleotides (5'-

TTCTCCGAACGTGTCACGT-3') and selection with $2.5 \mu \mathrm{g} / \mathrm{ml}$ puromycin or $10 \mu \mathrm{g} / \mathrm{ml}$ blasticidin. Antibioticresistant clones were picked and cultured in DMEM containing puromycin or blasticidin.

\section{Cytoplasmic and nuclear fractionation}

Harvested cells and tissues were extracted using the ProteoExtract ${ }^{\circledR}$ Subcellular Proteome Extraction Kit (539791, Millipore). The cytoplasmic and nuclear fractions were separated according to the manufacturer's instructions.

\section{Preparation of the hippocampal single-cell suspension}

The brain tissue was dissected on ice and enzymatically digested using the Adult Brain Dissociation kit (130-107-677, Miltenyi). The single-cell suspension was prepared for the flow cytometry assay according to the manufacturer's instructions.

\section{Flow cytometry}

Single-cell suspensions from each group were centrifuged and the pellet was resuspended with a viability dye Fixable Viability Stain 780 (1:1000; BD) for cell live/dead discrimination, followed by an incubation with blocking buffer containing CD16/CD32 (1:100; BD) for 10 min. Cells were then incubated with Foxp3/Transcription Factor Staining Buffer Set (00-5523, Invitrogen) at $4^{\circ} \mathrm{C}$ for $30 \mathrm{~min}$ in the dark. Finally, samples were incubated with the antibody mixture at $4{ }^{\circ} \mathrm{C}$ for $30 \mathrm{~min}$ in the dark, washed, centrifuged, resuspended in staining buffer containing $2 \%$ fetal bovine serum (FBS) and then sorted using a FACSAria SORP (BD, USA). Gating parameters and data analysis were performed using FlowJo 10 software (Tree Star, USA). The following antibodies were used for flow cytometry: mouse anti-SOX2 (Alexa Fluor 647) (1:100, Biolegend), mouse anti-DCX (PE) (1:100, BD), mouse anti-GFAP (Alexa Fluor 405) (1:100, Novus), rat anti-Ki67 (PerCP/Cy5.5) (1:100, Biolegend), mouse anti-AKT (Alxa Fluor 350) (1:100, R\&D Systems), and mouse anti-pAKT (S 473, BV421) (1:100, BD).

\section{EdU or BrdU incorporation assays}


For the analysis of newborn neuron proliferation in the hippocampus, the mice were injected with 50 mg/kg (i.p) bromodeoxyuridine (BrdU) (00-0103, Invitrogen) or 5-ethynyl-2'-deoxyuridine (Edu) (900584, Sigma) 3 times every $4 \mathrm{~h}$ on the last training day, and analyzed after the tests were complete [22]. BrdU and EdU immunostaining were performed to assess the proliferation of newborn hippocampal cells during cocaine memory formation.

For the flow cytometry assay of proliferation, single-cell suspensions were prepared from mice injected intraperitoneally with EdU as described above. Cultured N2a cells were serum-starved overnight, exposed to the external stimuli, and then $10 \mu \mathrm{M}$ EdU was added to the culture medium for $2 \mathrm{~h}$ before cells were collected.

For the flow cytometry detection of EdU expression, collected cells were transferred to 5-ml FACS tubes, fixed, washed with staining buffer and stained with the Click-iT ${ }^{\mathrm{TM}}$ Plus EdU Alexa Fluor ${ }^{\mathrm{TM}} 647$ Flow Cytometry Assay Kit (C10634, Thermo Scientific) at $4{ }^{\circ} \mathrm{C}$ for $30 \mathrm{~min}$ in the dark. Finally, samples were incubated with the antibody mixture at $4{ }^{\circ} \mathrm{C}$ for $30 \mathrm{~min}$ in the dark and then evaluated using a FACSAria SORP (BD, USA). Data were analyzed as described above.

\section{Quantification of the Tau-interacting proteome}

The dDG was removed from the hippocampus for co-IP, and the co-IP method was employed to detect the Tau-interacting proteome. The proteins were extracted and the co-IP procedures were conducted as described above. The collected protein supernatants were loaded and separated on 10\% sodium dodecyl sulfate-polyacrylamide gels, and then subjected to gel digestion followed by the LC-MS/MS analysis.

\section{In-gel digestion}

For in-gel tryptic digestion, gel pieces were destained in $50 \mathrm{mM} \mathrm{NH}_{4} \mathrm{HCO}_{3}$ in $50 \%$ acetonitrile (v/v) until they were clear. Gel pieces were dehydrated with $100 \mu$ l of $100 \%$ acetonitrile for 5 min, rehydrated in $10 \mathrm{mM}$ dithiothreitol and incubated at $56^{\circ} \mathrm{C}$ for $60 \mathrm{~min}$. Gel pieces were again dehydrated in $100 \%$ acetonitrile and rehydrated with $55 \mathrm{mM}$ iodoacetamide. Samples were incubated at room temperature for $45 \mathrm{~min}$ in the dark. Gel pieces were washed with $50 \mathrm{mM} \mathrm{NH}_{4} \mathrm{HCO}_{3}$ and dehydrated with $100 \%$ acetonitrile, followed by rehydration with $10 \mathrm{ng} / \mu$ trypsin and resuspension in $50 \mathrm{mM} \mathrm{NH}_{4} \mathrm{HCO}_{3}$ on ice for $1 \mathrm{~h}$. The excess liquid was removed and gel pieces were digested with trypsin at $37^{\circ} \mathrm{C}$ overnight. Peptides were extracted with $50 \%$ acetonitrile in $5 \%$ formic acid, followed by $100 \%$ acetonitrile. Finally, peptides were dried completely and resuspended in $2 \%$ acetonitrile in $0.1 \%$ formic acid.

\section{LC-MS/MS analysis}

The tryptic peptides were dissolved in $0.1 \%$ formic acid (solvent $A$ ), directly loaded onto a home-made reverse-phase analytical column (15-cm length, 75- $\mu \mathrm{m}$ i.d.). The gradient was comprised of $6-23 \%$ solvent $B(0.1 \%$ formic acid in $98 \%$ acetonitrile) in $16 \mathrm{~min}, 23-35 \%$ in $8 \mathrm{~min}$, increasing to $80 \%$ in $3 \mathrm{~min}$, and then maintained at $80 \%$ for the last 3 min with a constant flow rate of $400 \mathrm{nl} / \mathrm{min}$ on an EASY-nLC 1000 UPLC system. 
The peptides were subjected to an NSI source followed by tandem mass spectrometry (MS/MS) in Q Exactive $^{\text {TM }}$ Plus coupled online to the UPLC. The electrospray voltage applied was $2.0 \mathrm{kV}$. The $\mathrm{m} / \mathrm{z}$ scan range was 350 to 1800 for the full scan, and the intact peptides were detected in the Orbitrap at a 70000 resolution. Peptides were then selected for MS/MS using NCE at a setting of $28 \%$, and the fragments were detected in the Orbitrap at a resolution of 17500. A data-dependent procedure that alternated between one MS scan followed by $20 \mathrm{MS} / \mathrm{MS}$ scans with 15.0-s dynamic exclusion was performed. Automatic gain control (AGC) was set to $5 \mathrm{E} 4$.

\section{Data processing}

The MS/MS data were processed using Proteome Discoverer 1.3. Tandem mass spectra were searched in the SwissProt Mouse database. Trypsin/P was specified as the cleavage enzyme, allowing up to 2 missing cleavages. The mass error was set to $10 \mathrm{ppm}$ for precursor ions and $0.02 \mathrm{Da}$ for fragment ions. Carbamidomethylation on Cys was specified as the fixed modification and oxidation on Met was specified as the variable modification. Peptide confidence was set at high, and the peptide ion score was set to $>20$.

\section{Protein interaction networks}

All interesting gene name identifiers (mainly proteins involved in regulating cell proliferation) were searched against the STRING database version 10.5 for protein-protein interactions. Only interactions between the proteins belonging to the searched dataset were selected, thereby excluding external candidates. STRING defines a metric called a confidence score to define the interaction confidence, and we retrieved all interactions with a confidence score $\geq 0.7$ (high confidence). The interaction network identified using STRING was visualized with Cytoscape.

\section{Statistical analysis}

All data were analyzed with GraphPad Prism 7 software, presented as means \pm SEM, and subjected to the Kolmogorov-Smirnov test to assess the normality of the distribution. For simple comparisons, an unpaired two-tailed Student's $t$ test was used. For multiple comparisons, one-way or repeated-measures two-way ANOVA test was utilized for each experiment. In all cases, $n$ refers to the number of animals. For all results, statistical significance was defined as $p<0.05$.

\section{Results}

\section{Tau downregulation is involved in cocaine-cue memory formation}

Conditioned place preference (CPP) test, an associative memory model linking drug reward with environment cues, is widely used to assess the formation of drug-associated memory [23-25]. In the present study, we first investigated the effect of cocaine on the expression of Tau in the hippocampus during the cocaine memory formation. Compared with the control group, the mice trained with CPP 
showed significantly reduced level of Tau in the hippocampus (Fig. 1a-c). We next performed two other reward tests, the home cage CPP and food CPP tests, to investigate whether the changes in Tau level was specific to the formation of cocaine CPP memory. In the former test, mice were only injected with cocaine, but without cocaine-associated cue training (Fig. S1a, b). In the latter test, mice were only exposed to a palatable food reward to train the food-associated cue memory, but were not administered cocaine (Fig. S1d, e). Importantly, changes in the level of Tau was not observed in the hippocampus of the mice subjected to these two tests (Fig. S1c, f). We further measured Tau level after cocaine CPP extinction. Interestingly, the level of Tau clearly returned to baseline values (Fig. S1g-i).

We continued to investigate the role of Tau in cocaine self-administration, a paradigm that incorporates memories of drug experience and drug-associated environmental cues. Cocaine-associated memory was formed when an instrumental action (active pokes) resulted in cocaine delivery (unconditioned stimulus) paired with an audiovisual cue (conditioned stimulus). Mice were trained on a FR1 schedule, where a single active poke produced an infusion of cocaine or saline. Compared with saline-treated mice, cocaineself-administered mice showed reliable cocaine memory formation (Fig. 1d-g). Consistent with these findings, the level of Tau in the hippocampus was obviously reduced (Fig. 1h). We further detected Tau level in the hippocampus of cocaine-treated mice subjected to a locomotor activity test that lacks cocaine-associated contextual stimuli. Interestingly, Tau expression was not altered after cocaine injection for 7 days (Fig. 1i-k).

To further explore the role of Tau in cocaine memory formation, Tau-KO mice and non-transgenic wildtype (WT) mice were injected a low dose of cocaine $(2.5 \mathrm{mg} / \mathrm{kg}$ ) [26]. Notably, cocaine significantly induced a clear preference for the cocaine-paired chamber in Tau-KO mice that was not observed in WT mice (Fig.S2a-c). Collectively, Tau downregulation may play an important role in cocaine-associated memory formation.

\section{Tau downregulation promotes AHN during cocaine memory formation}

It has been known that AHN contributes to the formation of memory, including cocaine-associated memory $[12,13]$. To explore the role of Tau in AHN and cocaine memory formation, a flow cytometry assay was performed to investigate whether Tau ablation would affect the proliferation of newborn hippocampal neurons during cocaine memory formation. Notably, compared with WT mice, Tau-KO mice showed a qualitative increase in the proliferation of the neuronal subpopulations positive for SOX2 (a marker of neural stem cells and progenitor cells), SOX2/GFAP double-labelled cells (a marker of radial glia-like cells), and DCX (a marker of neuroblasts and immature neurons) (Fig. S2d-g). Thus, Tau deficiency may enhance AHN and facilitate cocaine memory formation.

\section{R Tau overexpression reduces AHN and attenuates cocaine memory formation}


To investigate whether Tau overexpression affects AHN and weakens cocaine memory formation, we first compared the expression of Tau isoforms in the dentate gyrus (DG), where AHN generally occurs [27]. Because the neuroanatomy of the hippocampus supports the segregation of neuronal outputs along a dorsal-ventral axis [28], the dorsal dentate gyrus (dDG) and ventral dentate gyrus (vDG) were separated from the hippocampus for immunoblotting analysis after CPP training, respectively. Both Tau and 4R Tau levels were significantly decreased in the dDG after cocaine CPP conditioning, whereas 3R Tau levels were not altered (Fig. S3a). In addition, the levels of the memory formation-related proteins phosphorylated CREB and phosphorylated cofilin were significantly increased; however, the levels of phosphorylated ERK remained unchanged (Fig. S3b). No significant differences in the levels of Tau, Tau isoforms, phosphorylated CREB or phosphorylated cofilin were observed in the VDG after cocaine CPP conditioning (Fig. S3c, d). Immunostaining further verified the decreased expression of both Tau and 4R Tau in the dDG of cocaine-conditioned mice (Fig. S3e-g). Collectively, 4R Tau, but not 3R Tau, may specifically modulate cocaine-induced memory formation.

We next explored the function of genetically overexpressed 4R Tau in AHN during cocaine memory formation. To this end, we constructed AAVs specifically expressing 2N4R Tau (AAV2/8-CMV-2N4R-2AmNeonGreen-3Flag), the longest brain 4R Tau isoform containing an intact Tau amino acid sequence [29]. AAV-2N4R Tau was stereotactically microinfused into the dDG before cocaine CPP training, and 2N4R Tau expression was visualized directly based on mNeonGreen expression (Fig. 2a). As expected, overexpression of AAV-2N4R Tau (AAV2/8-CMV-2N4R-3Flag) in the dDG significantly decreased the cocaine CPP score (Fig. 2b, c). Furthermore, the proliferation rates of the neuronal subpopulations positive for SOX2, both SOX2 and GFAP, and DCX were reduced (Fig. 2d-g). We further determined the effect of $4 R$ Tau overexpression on the proliferation of newborn hippocampal neurons in the dDG during cocaine memory formation. Mice were administered three consecutive injections of $\operatorname{BrdU}(50 \mathrm{mg} / \mathrm{kg}$, i.p.), a marker of the exogenous proliferation of newborn cells, in one day before the cocaine CPP test (Fig. 3a). Importantly, AAV-mediated 4R Tau overexpression markedly reduced the density of BrdUlabelled cells in the dDG of the hippocampus (Fig. 3b, c). In addition, the density of Ki67/BrdU doublelabelled cells was clearly reduced following the overexpression of 4R Tau (Fig. 3b, d). By quantifying the densities of SOX2/BrdU double-labelled cells (Fig. 3b, e), SOX2/GFAP/BrdU triple-labelled cells (Fig. 3b, f) and DCX/BrdU double-labelled cells (Fig. 3b, g), we found that 4R Tau exerted a deleterious effect on the proliferation of the aforementioned neuronal subpopulations. Furthermore, immunostaining and immunoblotting analyses revealed increased expression of the immediate early protein c-fos in the dDG during cocaine memory formation; nevertheless, this increase was obviously attenuated by 4R Tau overexpression (Fig. 3h-j). These results strongly support the hypothesis that 4R Tau overexpression inhibits the proliferation and activity of adult hippocampal neurons in the dDG during cocaine memory formation.

We continued to investigate the effect of $4 \mathrm{R}$ Tau overexpression on the levels of memory formationrelated proteins and the synaptic structure of cells in the granule layer of the $\mathrm{dDG}$. The phosphorylation levels of CREB and cofilin were obviously increased in WT mice after cocaine CPP paradigm; however, these increases were clearly attenuated by AAV-mediated 4R Tau overexpression. In contrast, the levels of 
total CREB and cofilin did not change (Fig. 4a, b). Golgi staining revealed a significant increase in the dendritic spine density of granule neurons after the cocaine CPP paradigm, whereas this increase was markedly inhibited by 4R Tau overexpression (Fig. 4c). In the cocaine self-administration model, the active pokes and numbers of cocaine infusions were significantly increased during cocaine memory formation; however, this increase was obviously attenuated by 4R Tau overexpression (Fig. 4d-g). Similarly, cocaine-induced increases in the levels of phosphorylated CREB and cofilin were decreased by 4R Tau overexpression (Fig. 4h, i). However, 4R Tau overexpression did not attenuate cocaine-induced hyperlocomotion in the absence of cocaine contextual stimuli (Fig. 4j-I). Thus, 4R Tau upregulation disrupts the formation of cocaine-associated memory and dendritic structural plasticity.

\section{R Tau modulates AHN by altering PI3K-AKT signaling during the cocaine memory formation}

Tau is able to bind to a variety of other proteins, including a number of proteins functioning in cell signaling transduction $[21,30]$. Tau-interacting proteome was analyzed to investigate the underlying mechanisms by which Tau regulates AHN. The dDG tissue was harvested, subjected to coimmunoprecipitation (co-IP), and analyzed using liquid chromatography coupled with tandem mass spectrometry (LC-MS/MS). Interestingly, 20 specific Tau-interacting proteins, which are intimately involved in regulating cell proliferation, were identified (Table S1). Eight of these proteins, such as PI3K-

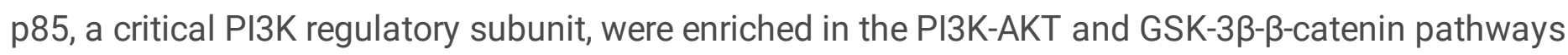
(Fig. 5a). Using an immunoblotting analysis, we confirmed that PI3K-AKT and GSK-3 $\beta$ - $\beta$-catenin signaling in the dDG were altered by cocaine CPP conditioning; however, AAV-mediated 4R Tau overexpression inhibited PI3K-AKT signaling, but not GSK-3ß-catenin signaling (Fig. 5b, c). We employed a co-IP analysis to further determine whether the interaction of Tau with PI3K-p85 would be altered by the cocaine CPP paradigm and found that both 3R Tau and 4R Tau bound endogenous PI3K-p85 in the dDG. Notably, the interaction of 4R Tau with PI3K-p85 was markedly reduced by cocaine CPP conditioning and reversed by the genetic overexpression of 4R Tau. However, the interaction of 3R Tau with PI3K-p85 was unchanged by cocaine CPP conditioning (Fig. 5d). Based on these results, the interaction of 4R Tau with PI3K-p85 may specifically modulate cocaine memory.

We subsequently performed immunofluorescence staining to track the cellular localization of these two proteins in the cultured N2a cells in vitro and to further clarify the interaction of 4R Tau with PI3K-p85. 4R Tau was primarily located in the cytosol of N2a cells and was clearly co-localized with PI3K-p85 (Fig.S4a). Co-IP assays further revealed the interaction of endogenous Tau with PI3K-p85 (Fig.S4b). An association of human Tau with PI3K-p85 has been shown in COS-7 cells, and the replacement of prolines 216 and 219 and arginine 221 of human Tau with alanine drastically decreased its binding to PI3Kp85[21]. By comparing the amino acid sequences of human and mouse Tau, prolines 216 and 219 and arginine 221 in human Tau correspond to prolines 205 and 208 and arginine 210 in mouse Tau, respectively. We then replaced these three amino acid residues of murine Tau with alanine and performed 
a co-IP assay. Notably, the interaction of mutant murine Tau with PI3K-p85 was markedly reduced (Fig.S4c), confirming the direct interaction of 4R Tau with PI3K-p85.

We further explored the function of the 4R Tau interaction with PI3K-p85 in the cultured N2a cells. N2a cells were serum-starved overnight, and then cultured with EdU, an exogenous proliferation marker, for $2 \mathrm{~h}$ before the cells were collected for flow cytometry detection. LV-mediated 4R Tau overexpression significantly inhibited the proliferation of N2a cells, whereas mutant 4R Tau exerted no effect (Fig.S4d). Moreover, the level of AKT phosphorylated at serine 473, a marker of PI3K-AKT activation, was markedly decreased by the overexpression of 4R Tau, but not mutant 4R Tau (Fig. S4e, f).

We further utilized a Tau shRNA to silence Tau expression in N2a cells and determined whether the downregulation of Tau expression promotes cell proliferation by reducing its association with PI3K-p85. The siRNA oligo pairs targeting Tau were transfected into N2a cells and efficiently knocked down Tau expression (Fig. 6a). The co-IP analysis revealed a decrease in the association of 4R Tau with PI3K-p85 (Fig. 6a). Not surprisingly, the Tau protein deficiency significantly increased N2a cell proliferation and the percentage of phosphorylated AKT-positive cells (Fig. 6b-d).

\section{R Tau restricts PI3K-p85 to the cytoplasm}

Because the nuclear accumulation of PI3K-p85 is required for PI3K-AKT signaling activity [31], we examined whether 4R Tau could trap endogenous PI3K-p85 to reduce its nuclear translocation in cells. Immunofluorescence staining revealed the even distribution of endogenous PI3K-p85 (red) in the cytosol and nucleus of N2a cells. Strikingly, endogenous PI3K-p85 was predominantly expressed in the nucleus when the Tau protein was deleted by a Tau shRNA (Fig. 6e). An exogenous PI3K-p85-expressing LV (red) was transfected into the N2a cells to further explore the effect of 4R Tau on the cellular distribution of PI3K-p85. Similarly, exogenously expressed PI3K-p85 was also mainly expressed in the cytoplasm. Importantly, the Tau-deficient cells exhibited a clear increase in the nuclear translocation of exogenous PI3K-p85 (Fig. 6f). We further separated cytosolic and nuclear fractions for western blot analysis. Tau deletion significantly increased the level of nuclear PI3K-p85 and the cytosolic level of phosphorylated AKT (Fig. 6g), suggesting that Tau, a cytoskeletal protein, may restrain PI3K-p85 in the cytoplasm, eventually blocking PI3K-AKT signaling. Besides, Tau-silenced N2a cells were pretreated with LY294002, a PI3K-AKT inhibitor, at a final concentration of $10 \mu \mathrm{M}$ to determine the role of PI3K-AKT signaling in N2a cell proliferation. The levels of PI3K-p110a and phosphorylated AKT were decreased by LY294002 in Tausilenced N2a cells (Fig. S5a). The flow cytometry analysis showed a clear decrease in the subpopulations of Tau-silenced N2a cells expressing both EdU and phosphorylated AKT (Fig. S5b-g). Based on these results, 4R Tau regulates PI3K-AKT signaling by binding to the PI3K-p85 subunit, subsequently modulating neuronal proliferation.

\section{The interaction of 4R Tau with PI3K-p85 modulates cocaine memory formation}


The 2N4R Tau-expressing AAV (AAV2/8-CMV-2N4R-2A-mNeonGreen-3Flag) was infused into the dDG of Tau-KO mice prior to the cocaine CPP paradigm to investigate the role of the 4R Tau and PI3K-p85 interaction in cocaine memory formation. Not surprisingly, 2N4R Tau overexpression significantly reduced the cocaine CPP score of Tau-KO mice; moreover, the co-IP assay showed an enhanced interaction of $4 \mathrm{R}$ Tau with PI3K-p85 (Fig. 7a-c). In Tau-KO mice, flow cytometry assays revealed a significant increase in the hippocampal cell populations labelled with EdU or antibodies against phosphorylated AKT; however, this effect was obviously reversed by AAV-mediated 2N4R Tau re-expression in the dDG (Fig. 7d-f). Similarly, the levels of nuclear PI3K-p85 and cytosolic phosphorylated AKT were also reduced by 2N4R Tau re-expression (Fig. 7g). However, re-expression of 2N4R Tau in the dDG did not reverse cocaineinduced hyperlocomotion in Tau-KO mice (Fig. 7h-j).

The mutant 2N4R Tau-expressing AAV (AAV2/8-CMV-mutant 2N4R-2A-mNeonGreen-3Flag) was stereotactically infused into the $\mathrm{dDG}$ of Tau-KO mice before cocaine CPP training. As expected, mutant 2N4R Tau exerted little effect on cocaine memory formation, and only a small amount of 2N4R Tau bound to PI3K-p85 (Fig. S6 a-c). Mutant 4R Tau also failed to reduce the number of the cells labelled with EdU or antibodies against phosphorylated AKT, or the nuclear PI3K-p85 levels and cytosolic phosphorylated AKT levels (Fig. S6d-g).

Finally, we applied LY294002 to pharmacologically suppress PI3K-AKT signaling in the dDG of Tau-KO mice. LY294002 was micro-infused into the dDG of mice through an implanted cannula before CPP training (Fig. S7a). As expected, the mice pretreated with LY294002 displayed a significant decrease in the cocaine CPP score (Fig.S7b, c). In addition, cocaine-induced changes in the levels of PI3K-p110a and phosphorylated AKT were attenuated by LY294002 (Fig.S7d). The proliferation rates of the neuronal subpopulations labelled with Ki67/SOX2 and Ki67/DCX were also reduced by the LY294002 treatment (Fig. S7e, f).

In summary, downregulation of $4 \mathrm{R}$ Tau reduces its association with PI3K-p85 and thus promotes PI3Kp85 nuclear translocation to activate PI3K-AKT signaling, which eventually promotes AHN and evokes cocaine-cue memory formation.

\section{Discussion}

Tau accumulation plays an important role in memory impairment [32,33], and a decrease in Tau expression prevents neuronal loss, amyloid beta-induced axonal transport deficits as well as memory impairment in tauopathy models [34-36]. In the present study we found a decreased expression of hippocampal Tau during cocaine memory formation; however, Tau expression remained unchanged in response to non-cocaine-conditioned stimuli. Furthermore, 4R Tau overexpression in the dDG disrupted cocaine memory formation, hippocampal neuronal proliferation and activity, and cocaine-seeking behaviors. In addition, cocaine-induced downregulation of 4R Tau clearly increased the expression of phosphorylated CREB, which is preferentially recruited or allocated to cocaine engram cells to encode 


\section{Distinct functions of Tau isoforms in the DG during cocaine memory formation}

The segregation of neuronal outputs along the dorsal-ventral axis and their connectivity affect neurogenesis in the DG and hippocampus-associated memory [38]. During the cocaine contextual memory formation, 4R Tau expression was reduced in the $\mathrm{dDG}$, but not in the vDG. The difference observed in these two brain regions may be attributed to the functional dissociation that exists along the dorsal-ventral gradient in the hippocampus [28]. Indeed, the dorsal hippocampus is more important for spatial learning and contextual discrimination than the ventral region [38, 39]. In contrast, the ventral hippocampus is strongly associated with negative affective symptoms, such as emotional behavior and stress responses [40].

The functional neuronal expression of Tau isoforms has been a subject of controversy and debate. During brain development, the expression of 3R Tau and 4R Tau isoforms shifts during AHN [41], and 3R Tau provides a dynamic microtubule network in DCX-positive cells, allowing proper axonal growth during AHN [42]. Interestingly, cocaine decreased hippocampal 4R Tau expression, but had little effect on 3R Tau expression, suggesting different functions of Tau isoforms in specific neuron populations. Indeed, 3R Tau binds with a lower affinity to microtubules, potentially contributing to the cytoskeletal plasticity. In contrast, 4R Tau is mainly expressed in the adult brain, exhibits higher affinity for microtubules, and functions in the establishment and maintenance of the synaptic structure of newly integrated neurons [20]. Therefore, based on our findings, 4R Tau may contribute to the structural remodeling and integration of newborn neurons into the hippocampal circuit in response to drug conditioning.

\section{R Tau regulates cocaine-associated memory by modulating AHN}

AHN is also a process in which adult-born hippocampal neurons are functionally integrated into the addiction network by interacting with addiction-related brain regions to contribute to cocaine memory formation [11]. In our study, AHN in the dDG was tightly regulated by 4R Tau during cocaine memory formation, and a reduction in cell proliferation inhibited memory formation in both the cocaine-paired CPP and self-administration paradigms. Intriguingly, a few studies reported that cocaine is a potent suppressor of $A H N$ and might frequently reduce cell proliferation in rodents $[43,44]$. However, these results were obtained from experiments in which rodents were forcibly administered cocaine in their home cage; moreover, the measurement of cell proliferation was usually performed immediately or soon after cocaine administration in an open-field environment [45]. More importantly, these experiments lacked the cocaine-associated contextual stimuli that is critical for cocaine-cue memory formation. 
AHN induced by a genetic, pharmacological or environmental approach is able to exacerbate cocaineseeking behavior, showing a role of $A H N$ in increasing the vulnerability to the action of drugs $[12,13,46]$. For example, through reducing $A H N$, mice exhibited higher motivation to cocaine self-administration and drug-seeking behaviors in the phase of reinstatement [46]. On the contrary, exercise before CPP training, which increases AHN, promotes cocaine memory formation [14]. Therefore, we infer that that if cocaine CPP conditioning is conducted in the animals with increased numbers of adult-born hippocampal neurons, more young neurons might be recruited for CPP training. It would explain why the cocaineassociated memory is more easily formed after increasing AHN [14]. Accordingly, reducing AHN in the acquisition phase of cocaine memory formation may suppress the survival, maturation, and/or function integration of the new neurons involved in the memory circuits and eventually impair cocaine memory formation. However, once cocaine memory has been formed, such as in cocaine withdrawal phase, reducing AHN may fail to weaken the previous cocaine memory, which trigger animal drug seeking and relapse during the period of abstinence [46]. Our findings highlight the existence of a dynamic population of proliferating neurons in the $D G$, and $A H N$ clearly emerges as a robust phenomenon during the cocaine memory formation.

\section{The interaction of 4R Tau with PI3K-p85 modulates PI3K- AKT signaling}

By analyzing the Tau-interacting proteome, we revealed that PI3K-p85 is the key regulator of Taumediated AHN. Although accumulating studies have shown that PI3K-AKT signaling regulates hippocampal neuron survival and proliferation in the DG upon exposure to external chronic stress, the role of PI3K-AKT signaling in cocaine memory formation has not been completely elucidated $[17,47]$. Only one study published to date reported an association of human Tau with PI3K-p85 in COS-7 cells, and the replacement of a few amino acid residues of human Tau drastically decreased its binding with PI3K-p85 [21]. Here, we provide strong evidence that 4R Tau directly binds to PI3K-p85 and may restrict its nuclear translocation, thus modulating PI3K-AKT signaling. In turn, the cocaine-induced downregulation of $4 \mathrm{R}$ Tau may diminish the cytoplasmic localization of PI3K-p85 and subsequently increase its nuclear transportation, which is essential for cell proliferation and AHN.

In fact, as a critical regulatory subunit, PI3K-p85 interacts with several proteins to regulate the activation of the PI3K-AKT signaling pathway. For instance, the interaction of PI3K-p85 with bromodomaincontaining protein 7 (BRD7) [31], C-type lectin-like receptor 2 or leucine zipper tumor suppressor 2 suppresses PI3K-AKT signaling [48, 49]. In contrast, the interaction of PI3K-p85 with CD133 activates PI3K-AKT signaling [50]. Therefore, through its interacting proteins, PI3K-p85 appears to exert distinct functions in the regulation of PI3K-AKT signaling. In the present study, the cellular distribution of PI3Kp85 was tightly regulated by 4R Tau, and the 4R Tau deficiency induced PI3K-p85 nuclear translocation, thus promoting neuronal proliferation. Further studies are needed to investigate the nuclear transport dynamics of PI3K-p85 and its precise function in neuronal proliferation during cocaine memory formation. 


\section{Conclusions}

Collectively, 4R Tau regulates cocaine-associated memory through an AHN-dependent mechanism. This finding suggests the presence of unexplored mechanisms of cocaine-induced circuit plasticity in the hippocampus, and may have implications for the development of novel therapeutic strategies aimed at restoring a normal level of $\mathrm{AHN}$ or the disruption of the Tau-mediated synaptic plasticity that occurs in response to cocaine.

\section{Abbreviations}

3R Tau: Three microtubule-binding repeats Tau; 4R Tau: Four microtubule-binding repeats Tau; AAVs: Adeno-associated viruses; AHN: Adult hippocampal neurogenesis; BrdU: Bromodeoxyuridine; BRD7: Bromodomain-containing protein 7; CPP: Conditioned place preference; Co-IP: Co-immunoprecipitation; DG: Dentate gyrus; dDG: Dorsal dentate gyrus; Edu: Ethynyl-2'-deoxyuridine; FR1: Fixed ratio 1; HE: Hematoxylin eosin; PI3K: Phosphoinositide 3-kinase; Tau-KO: Tau ${ }^{-1-}$ knockout; vDG: ventral dentate gyrus; WT: Wild-type

\section{Declarations}

\section{Ethics approval and consent to participate}

All animal experiments were conducted with the approval of the Institutional Animal Care and Use Committee of Sichuan University.

\section{Consent for publication}

All authors have approved of the contents of this manuscript and provided consent for publication.

\section{Availability of data and materials}

All data needed to evaluate the conclusions in the paper are present in the paper and/or the Supplementary Materials. Additional data related to this paper may be requested from the authors.

\section{Competing interests}

The author declare that they have no competing interests.

\section{Funding}

This work was partially supported by the National Natural Science Foundation of China (Grants 81871043, 81571301 and 81401105), National Science and Technology Major Project (2018ZX09201017 
and 2018ZX09201018), and 1.3.5 Project for Disciplines of Excellence, West China Hospital, Sichuan University (ZYGD18024).

\section{Author contributions}

H.L., W.X.: conception and design, performed the experiments, acquisition of data as well as analysis and interpretation of data, and wrote the manuscript; L.W., C.L., Q.F., Y.H., X.L., J.Z., X.W., X.Y., Q.B., H.W., J.T. and Y.Z.: acquisition of data as well as analysis and interpretation of data; D.W.: conception and design of cell experiments; H.L., J.Z. and Z.Y.: analysis and interpretation of flow cytometry and immunostaining data; B.C.: conceived and supervised this research, as well as drafted and revised the article. All authors read and approved the final manuscript $\square$

\section{Acknowledgments}

We would like to acknowledge professor Peng Lei, State Key Laboratory of Biotherapy in West China hospital of Sichuan University, for providing Tau-KO mice.

\section{Author details}

${ }^{1}$ National Chengdu Center for Safety Evaluation of Drugs, State Key Laboratory of Biotherapy and Cancer Center, West China Hospital, Sichuan University, and Collaborative Innovation Center for Biotherapy, 610041 Chengdu, China

${ }^{2}$ Department of Respiratory and Critical Care Medicine, State Key Laboratory of Biotherapy and Cancer Center, West China Hospital, Sichuan University, 610041 Chengdu, China

${ }^{3}$ Research Core Facility, West China Hospital, Sichuan University, 610041 Chengdu, China

${ }^{4}$ Ministry of Education, Collaborative Innovation Center of Advanced Drug Delivery System and Biotech Drugs in Universities of Shandong, Yantai University, Yantai 264005, China.

\section{References}

1. Wolf ME. Synaptic mechanisms underlying persistent cocaine craving. Nature reviews Neuroscience. 2016;17(6):351-65.

2. Liddie $S$, Itzhak Y. Variations in the stimulus salience of cocaine reward influences drug-associated contextual memory. Addict Biol. 2016;21(2):242-54.

3. Castilla-Ortega E, et al. Pharmacological reduction of adult hippocampal neurogenesis modifies functional brain circuits in mice exposed to a cocaine conditioned place preference paradigm. Addict Biol. 2016;21(3):575-88.

4. Liu C, et al. Retrieval-Induced Upregulation of Tet3 in Pyramidal Neurons of the Dorsal Hippocampus Mediates Cocaine-Associated Memory Reconsolidation. The international journal of 
neuropsychopharmacology. 2018;21(3):255-66.

5. Deschaux 0, et al. Hippocampal neurogenesis protects against cocaine-primed relapse. Addict Biol. 2014;19(4):562-74.

6. Tobin MK, et al. Human Hippocampal Neurogenesis Persists in Aged Adults and Alzheimer's Disease Patients. Cell Stem Cell. 2019;24(6):974-82. e973.

7. Moreno-Jimenez EP, et al. Adult hippocampal neurogenesis is abundant in neurologically healthy subjects and drops sharply in patients with Alzheimer's disease. Nature medicine. 2019;25(4):55460.

8. Takashima Y, Mandyam CD. The role of hippocampal adult neurogenesis in methamphetamine addiction. Brain plasticity. 2018;3(2):157-68.

9. Toda T, Gage FH. Review: adult neurogenesis contributes to hippocampal plasticity. Cell tissue research. 2017;373(3):693-709.

10. Lazarov O, Hollands C. Hippocampal neurogenesis: Learning to remember. Progress in neurobiology. 2016;138-140:1-18.

11. Castilla-Ortega $E$, et al. A place for the hippocampus in the cocaine addiction circuit: Potential roles for adult hippocampal neurogenesis. Neuroscience Biobehavioral Reviews. 2016;66:15-32.

12. Castilla-Ortega $E$, et al. The impact of cocaine on adult hippocampal neurogenesis: Potential neurobiological mechanisms and contributions to maladaptive cognition in cocaine addiction disorder. Biochem Pharmacol. 2017;141:100-17.

13. Castilla-Ortega E, Santin LJ. Adult hippocampal neurogenesis as a target for cocaine addiction: a review of recent developments. Curr Opin Pharmacol. 2019.

14. Mustroph ML, Stobaugh DJ, Miller DS, DeYoung EK, Rhodes JS. Wheel running can accelerate or delay extinction of conditioned place preference for cocaine in male C57BL/6J mice, depending on timing of wheel access. Eur J Neurosci. 2011;34(7):1161-9.

15. Guo T, Noble W, Hanger DP. Roles of tau protein in health and disease. Acta Neuropathol. 2017;133(5):665-704.

16. Criado-Marrero M, et al. Hippocampal Neurogenesis Is Enhanced in Adult Tau Deficient Mice. Cells. 2020; 9(1).

17. Dioli $\mathrm{C}$, et al. Tau-dependent suppression of adult neurogenesis in the stressed hippocampus. Mol Psychiatry. 2017;22(8):1110-8.

18. Pallas-Bazarra N, et al. Novel function of Tau in regulating the effects of external stimuli on adult hippocampal neurogenesis. The EMBO journal. 2016;35(13):1417-36.

19. Tuerde D, et al. Isoform-independent and -dependent phosphorylation of microtubule-associated protein tau in mouse brain during postnatal development. J Biol Chem. 2018;293(5):1781-93.

20. M L \& KS K. Competition for microtubule-binding with dual expression of tau missense and splice isoforms. Molecular biology of the cell. 2001; 12(1):171-184. 
21. Reynolds $\mathrm{CH}$, et al. Phosphorylation regulates tau interactions with Src homology 3 domains of phosphatidylinositol 3-kinase, phospholipase Cgamma1, Grb2, and Src family kinases. J Biol Chem. 2008;283(26):18177-86.

22. Wang J, et al. Brain Endothelial Cells Maintain Lactate Homeostasis and Control Adult Hippocampal Neurogenesis. Cell Stem Cell. 2019;25(6):754-67 e759.

23. Y Z, et al. A ventral CA1 to nucleus accumbens core engram circuit mediates conditioned place preference for cocaine. Nature neuroscience. 2019; 22(12):1986-1999.

24. Bardo MT, Bevins RA. Conditioned place preference: what does it add to our preclinical understanding of drug reward? Psychopharmacology. 2000;153(1):31-43.

25. Wang Z, et al. Retrieval-Driven Hippocampal NPTX2 Plasticity Facilitates the Extinction of CocaineAssociated Context Memory. Biol Psychiatry. 2020;87(11):979-91.

26. Salery M, et al. Activity-Regulated Cytoskeleton-Associated Protein Accumulates in the Nucleus in Response to Cocaine and Acts as a Brake on Chromatin Remodeling and Long-Term Behavioral Alterations. Biol Psychiatry. 2017;81(7):573-84.

27. Altman J, Das GD. Autoradiographic and histological evidence of postnatal hippocampal neurogenesis in rats. J Comp Neurol. 1965;124(3):319-35.

28. Fanselow MS, Dong HW. Are the dorsal and ventral hippocampus functionally distinct structures? Neuron. 2010;65(1):7-19.

29. Avila J, et al. Tau Structures Frontiers in aging neuroscience. 2016;8:262.

30. R B \& J L. Functional interactions of tau and their relevance for Alzheimer's disease. Current Alzheimer research. 2004; 1(4):255-269.

31. Chiu YH, Lee JY, Cantley LC. BRD7, a tumor suppressor, interacts with p85alpha and regulates PI3K activity. Molecular cell. 2014;54(1):193-202.

32. Li XG, et al. Tau accumulation triggers STAT1-dependent memory deficits by suppressing NMDA receptor expression. EMBO Rep. 2019;20(6):e47202.

33. Yin Y, et al. Tau accumulation induces synaptic impairment and memory deficit by calcineurinmediated inactivation of nuclear CaMKIV/CREB signaling. Proceedings of the National Academy of Sciences. 2016; 113(26):E3773-3781.

34. DeVos SL, et al. Tau reduction prevents neuronal loss and reverses pathological tau deposition and seeding in mice with tauopathy. Sci TransI Med. 2017; 9(374).

35. Vossel KA, et al. Tau reduction prevents $A \beta$-induced axonal transport deficits by blocking activation of GSK3 3 . The Journal of Cell Biology. 2015;209(3):419-33.

36. Vossel KA, et al. Tau reduction prevents Abeta-induced defects in axonal transport. Science. 2010;330(6001):198.

37. Hsiang HL, et al. Manipulating a "cocaine engram" in mice. The Journal of neuroscience: the official journal of the Society for Neuroscience. 2014;34(42):14115-27. 
38. Pierard C, Dorey R, Henkous N, Mons N, Beracochea D. Different implications of the dorsal and ventral hippocampus on contextual memory retrieval after stress. Hippocampus. 2017;27(9):9991015.

39. Wells AM, et al. Contribution of an SFK-Mediated Signaling Pathway in the Dorsal Hippocampus to Cocaine-Memory Reconsolidation in Rats. Neuropsychopharmacology. 2015;41(3):675-85.

40. Anacker $\mathrm{C}$, et al. Hippocampal neurogenesis confers stress resilience by inhibiting the ventral dentate gyrus. Nature. 2018;559(7712):98-102.

41. Fuster-Matanzo A, Llorens-Martin M, Jurado-Arjona J, Avila J, Hernandez F. Tau protein and adult hippocampal neurogenesis. Front NeuroSci. 2012;6:104.

42. Llorens-Martin $\mathrm{M}$, et al. Tau isoform with three microtubule binding domains is a marker of new axons generated from the subgranular zone in the hippocampal dentate gyrus: implications for Alzheimer's disease. Journal of Alzheimer's disease: JAD. 2012;29(4):921-30.

43. Sudai $E$, et al. High cocaine dosage decreases neurogenesis in the hippocampus and impairs working memory. Addict Biol. 2011;16(2):251-60.

44. GarcÃa-Fuster MJ, Perez JA, Clinton SM, Watson SJ, Akil H. Impact of cocaine on adult hippocampal neurogenesis in an animal model of differential propensity to drug abuse. Eur $\mathrm{J}$ Neurosci. 2010;31(1):79-89.

45. Xie LL, et al. Aquaporin 4 knockout resists negative regulation of neural cell proliferation by cocaine in mouse hippocampus. The international journal of neuropsychopharmacology. 2009;12(6):84350.

46. Deroche-Gamonet V, et al. Depleting adult dentate gyrus neurogenesis increases cocaine-seeking behavior. Mol Psychiatry. 2019;24(2):312-20.

47. Tiwari SK, et al. Ethosuximide Induces Hippocampal Neurogenesis and Reverses Cognitive Deficits in an Amyloid-beta Toxin-induced Alzheimer Rat Model via the Phosphatidylinositol 3-Kinase (PI3K)/Akt/Wnt/beta-Catenin Pathway. J Biol Chem. 2015;290(47):28540-58.

48. Wang L, et al. C-Type Lectin-Like Receptor 2 Suppresses AKT Signaling and Invasive Activities of Gastric Cancer Cells by Blocking Expression of Phosphoinositide 3-Kinase Subunits. Gastroenterology. 2016;150(5):1183-95.e1116.

49. Xu S, et al. LZTS2 inhibits PI3K/AKT activation and radioresistance in nasopharyngeal carcinoma by interacting with p85. Cancer letters. 2018;420:38-48.

50. Wei Y, et al. Activation of PI3K/Akt pathway by CD133-p85 interaction promotes tumorigenic capacity of glioma stem cells. Proceedings of the National Academy of Sciences. 2013; 110 (17):6829-6834.

\section{Figures}




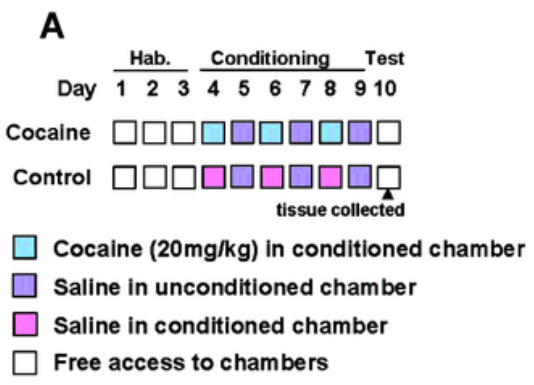

D

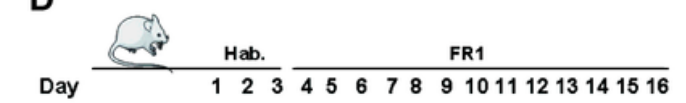

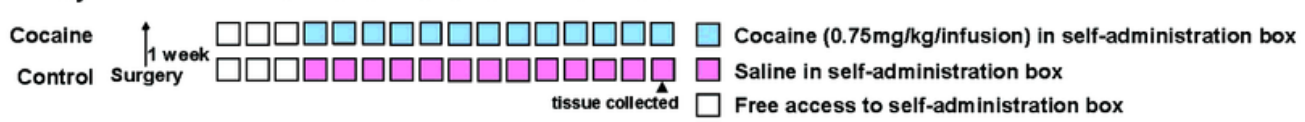

F
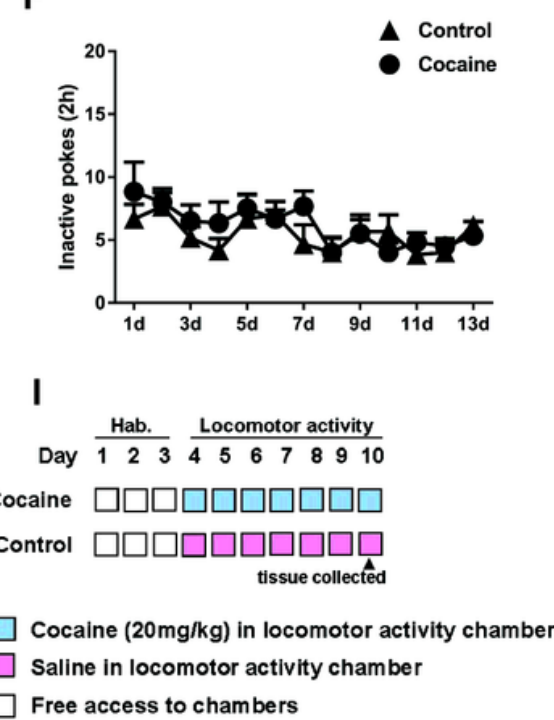

Hab. Locomotor activity

cocaine

Control $\square \square \square \square \square \square \square \square \square \square$

Saline in locomotor activity chamber

Free access to chambers
B

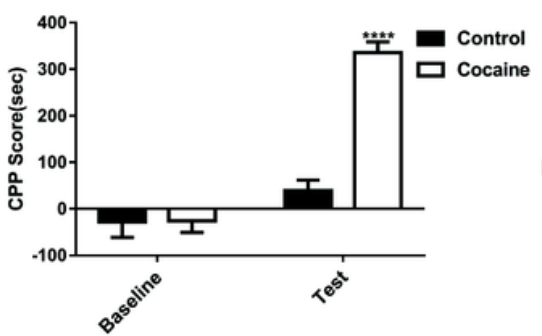

G

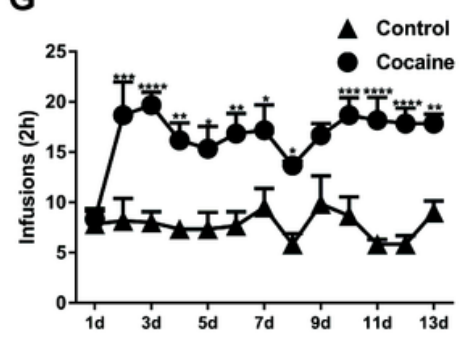

J

C

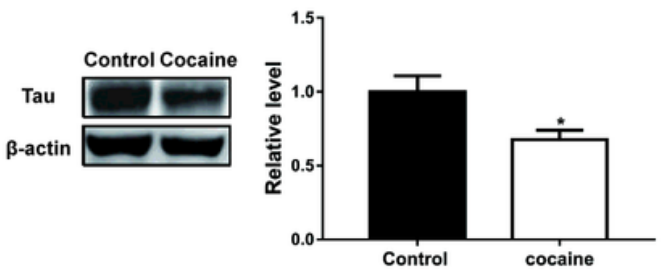

E
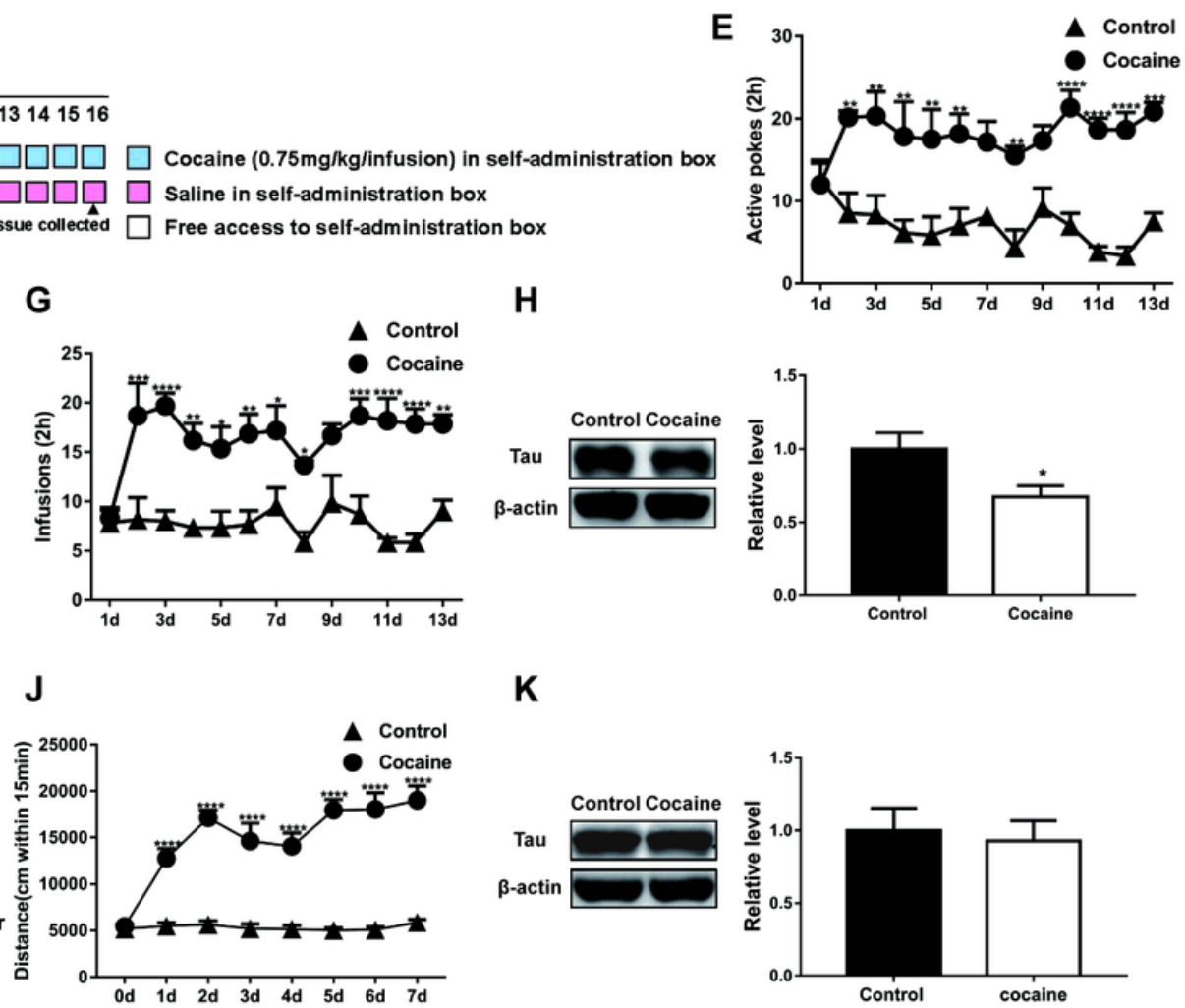

Tau

Control Cocaine

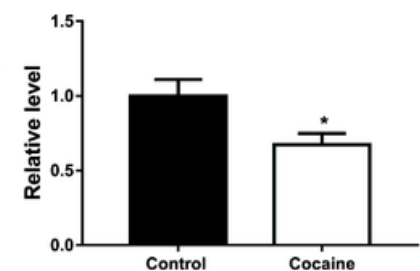

K

H
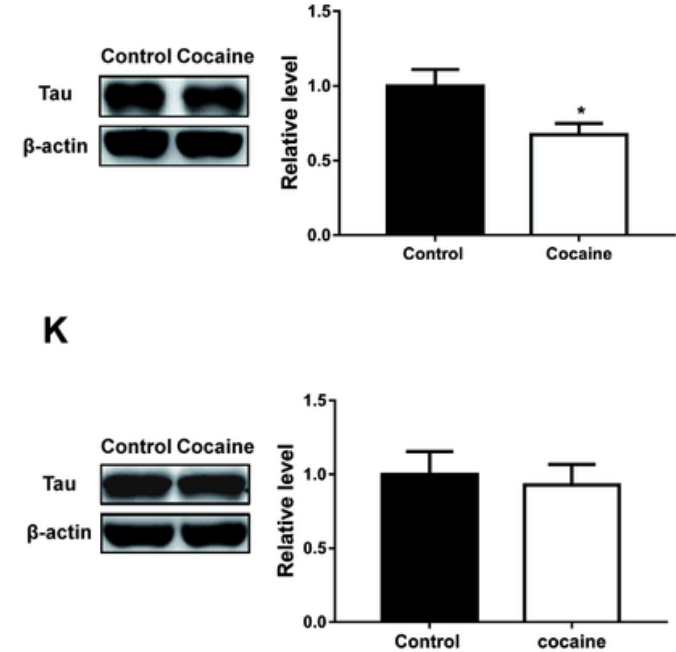

\section{Figure 1}

Cocaine-cue memory downregulates the expression of Tau and phosphorylated Tau in the hippocampus. a Experimental schedule for cocaine CPP. b Cocaine CPP score was significantly increased in cocaine group when compared to control group ( $\mathrm{n}=15$ per group). c Expression of Tau and phosphorylated Tau in the hippocampus with cocaine CPP conditioning ( $n=8$ per group). $d$ Timeline of cocaine selfadministration experiment. (e-g) Number of active pokes, inactive pokes and infusions in the cocaine selfadministration ( $n=6$ per group). $h$ Expression of Tau and phosphorylated Tau in the hippocampus of cocaine self-administered mice ( $n=6$ per group). i Schematic of the locomotor-activity procedure. $j$ Distance during the 15 min after daily cocaine administration ( $n=12$ per group). $k$ Expression of Tau and phosphorylated Tau in the hippocampus in cocaine-induced hyperlocomotion ( $\mathrm{n}=8$ per group). Data are the means \pm SEM, ${ }^{*} p<0.05 ;{ }^{* *} p<0.01 ; * \star * p<0.001$ and ${ }^{* \star * \star} p<0.0001$. Hab, habituation; $p$, phosphorylated; S, Serine; T, tyrosine. Tau phosphorylation at the residues of 199 serine (pS 199), 231 tyrosine (pT 231), 396 serine (pS 396) and 404 serine (pS 404). 
A

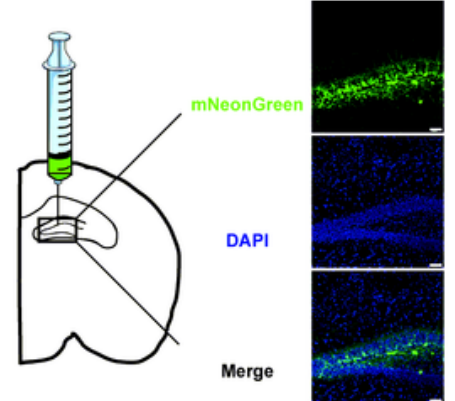

B

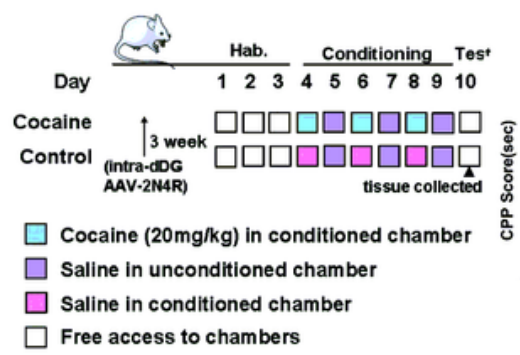

C

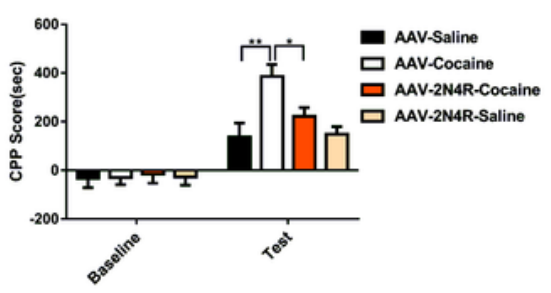

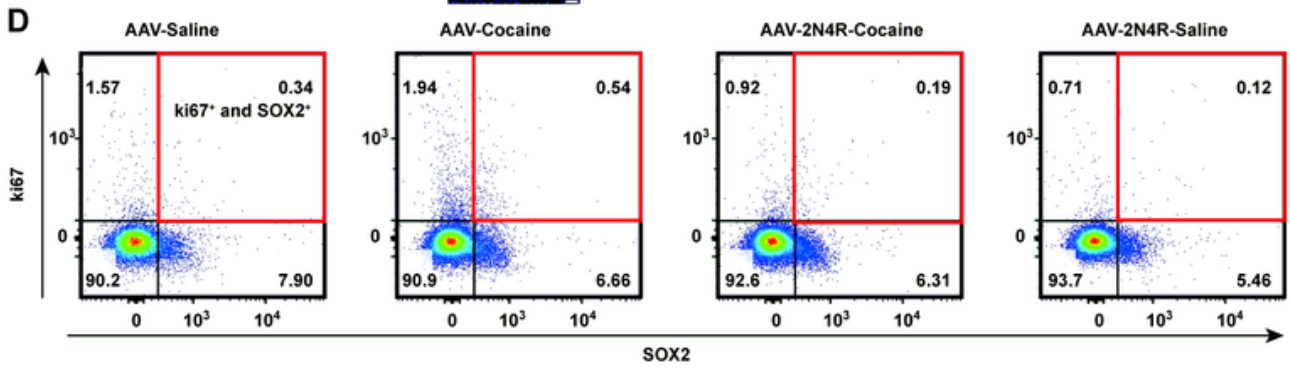

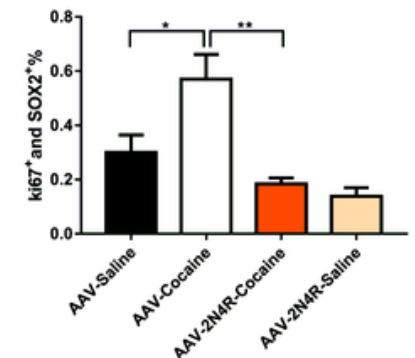

E
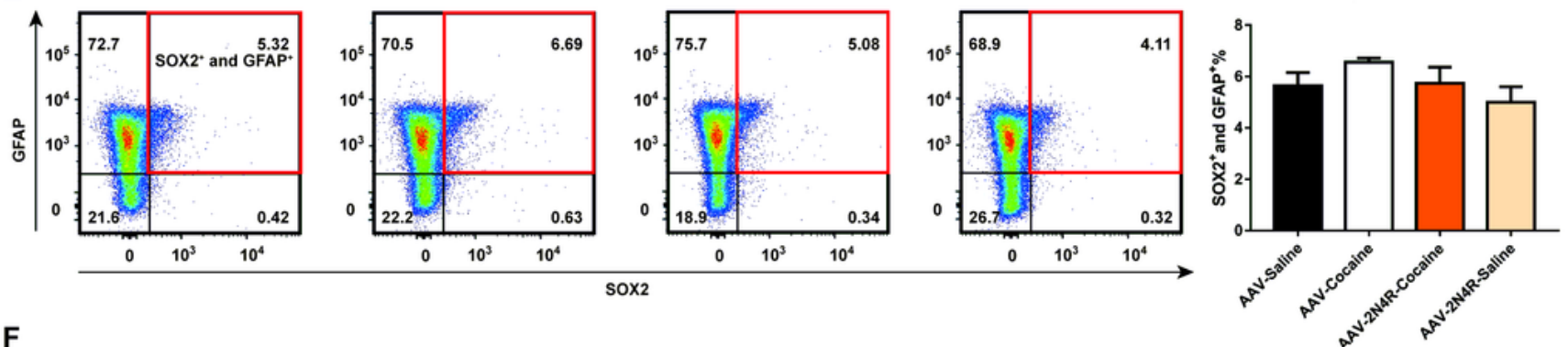

F
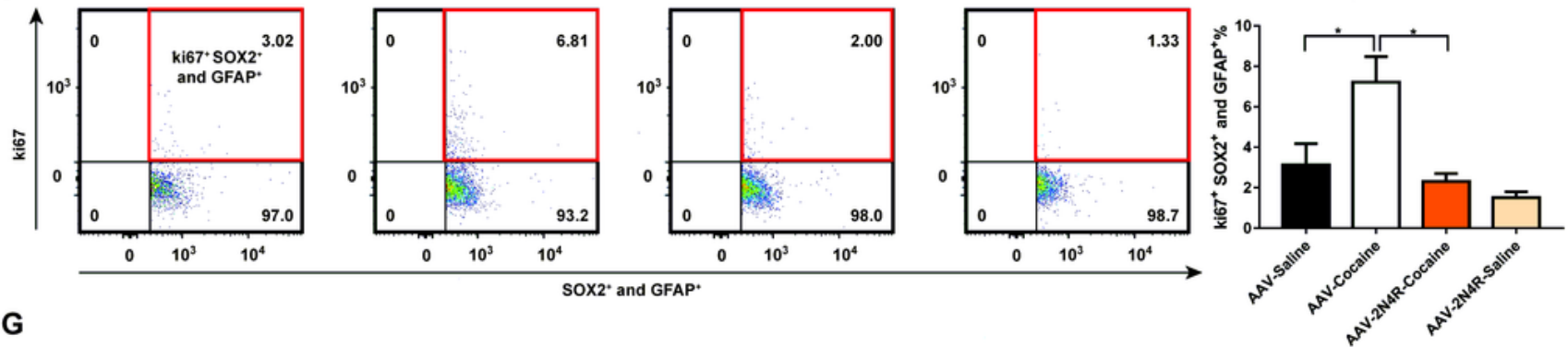

G
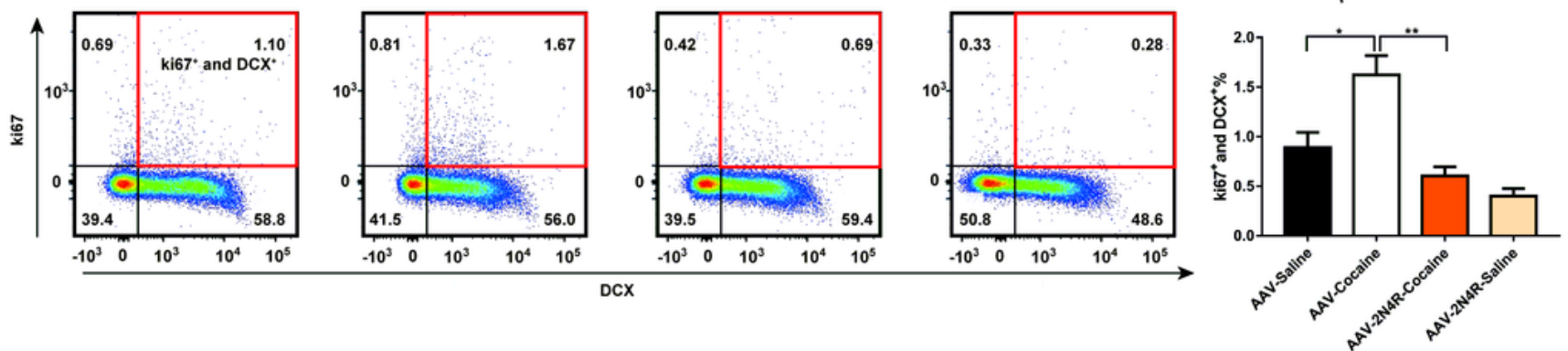

Figure 2

AAV-mediated 4R Tau overexpression attenuates the proliferation of adult hippocampal neurons during cocaine memory formation. a Representative images of AAV2/8-mNeonGreen-2N4R Tau expression in the dDG (green); DAPI, nucleus (blue). b Experiment timeline for AAV-mediated 4R Tau overexpression in the dDG 3 weeks prior to cocaine CPP training. c AAV-mediated 4R Tau overexpression in the dDG weakened cocaine-induced CPP ( $\mathrm{n}=12$ per group). (d-g) Representative flow cytometry plots depict the effect of AAV- 
mediated 4R Tau overexpression on the proliferation rates of hippocampal neuron. The upper right quadrants show the corresponding positive subpopulations ( $n=4$ AAV-saline group, $n=3$ AAV-cocaine group, $n=3$ AAV-2N4R-cocaine group, $n=3$ AAV-2N4R-saline group). Data are the means $\pm S E M,{ }^{*} p<0.05$ and $* * p<0.01$.

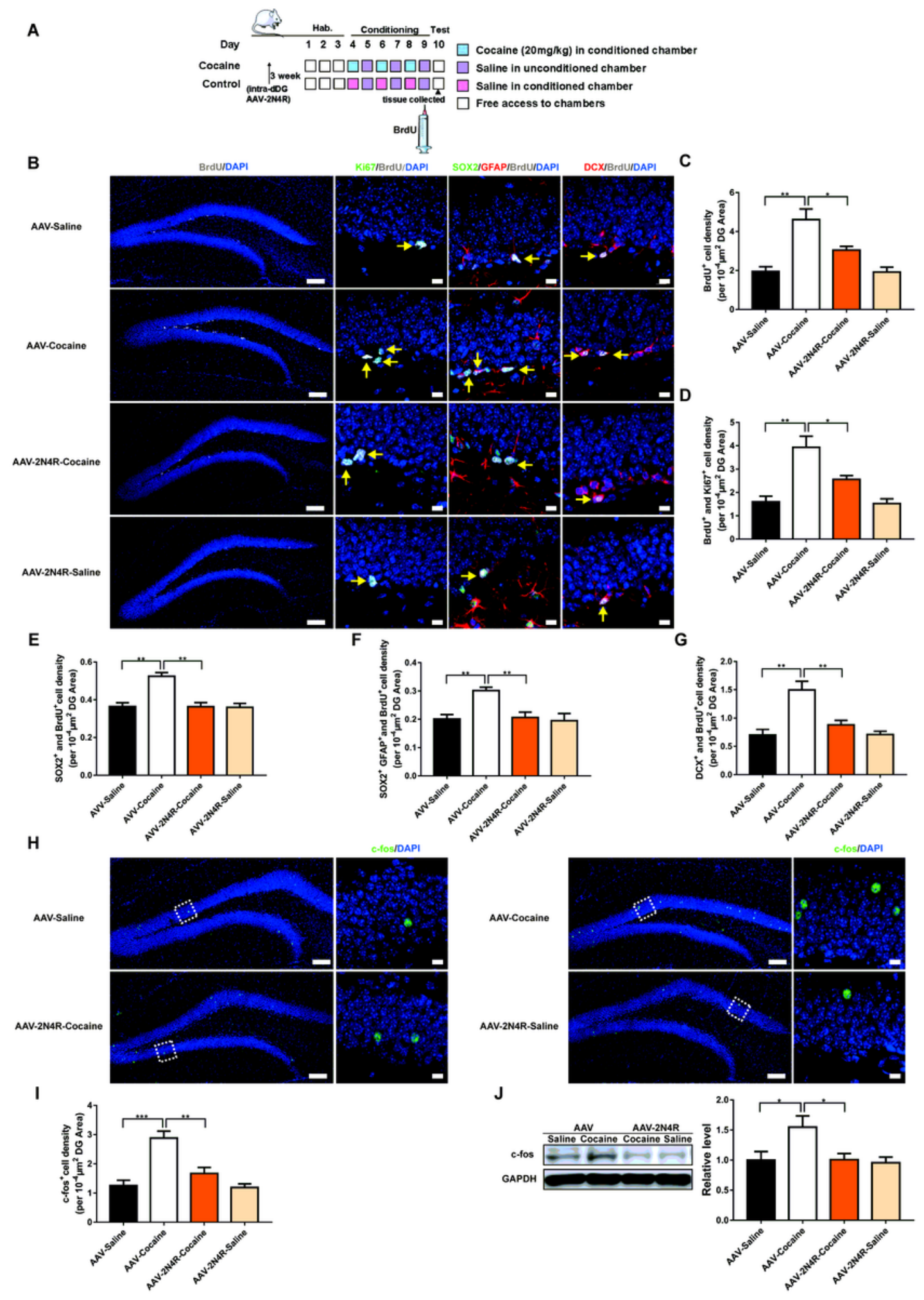

Figure 3 
AAV-mediated 4R Tau overexpression suppresses cocaine CPP-promoted proliferating rates and activity of hippocampal neuron. a Schematic representation of experiment timeline for mice receiving BrdU injection. (b-d) The density of BrdU and BrdU/Ki67 double-labelled cells in the dDG was reduced by AAVmediated 4R Tau overexpression ( $n=3$ per group). $b$, e The rate of SOX2/BrdU double-labelled cells was reduced by AAV-mediated 4R Tau overexpression ( $n=3$ per group). $b$, $f$ Confocal images showed a decreased density of SOX2/ GFAP/ BrdU triple-labelled cells in the dDG after AAV-mediated 4R Tau overexpression ( $n=3$ per group). $b, g$ The density of DCX/BrdU double-labelled cells were reduced by AAVmediated 4R Tau overexpression ( $n=3$ per group). $h$, i Confocal images of $c-f o s$ immunostaining and quantification showed a decreased density of c-fos labelled cells in dDG by AAV-mediated 4R Tau overexpression ( $n=3$ per group). $j$ Immunoblotting for $c-f o s$ in the dDG ( $n=6$ per group). The long scale bars for $100 \mu \mathrm{m}$; the short scale bars for $10 \mu \mathrm{m}$. Data are the means $\pm S E M,{ }^{*} p<0.05,{ }^{\star \star} p<0.01$ and $\star * * \mathrm{p}<0.001$. 
A
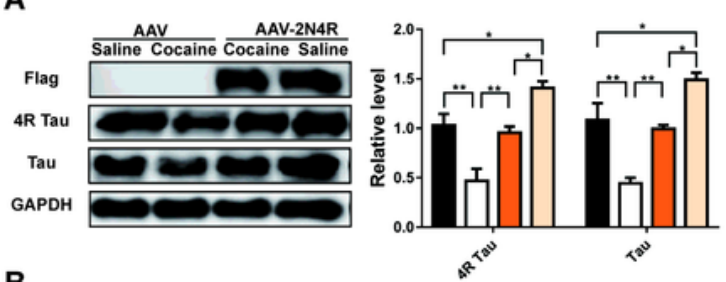

B
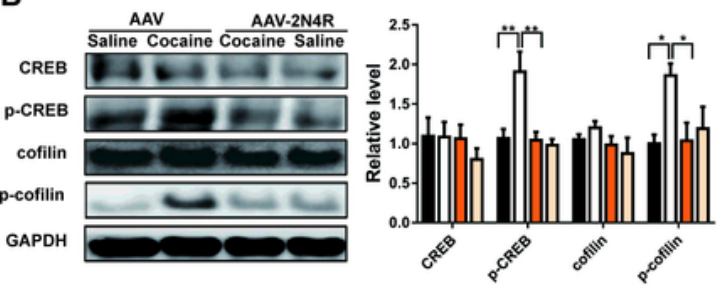

D

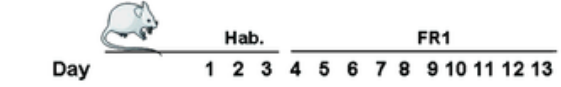

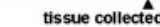

$\square$ Cocaine $(0.75 \mathrm{mg} / \mathrm{kg} /$ infusion) in self-administration box

$\square$ Saline in self-administration box

$\square$ Free access to box

- AAV-Saline

口 AAV-Cocaine

口AAV-2N4R-Cocain

口 AAV-2N4R-Saline

C
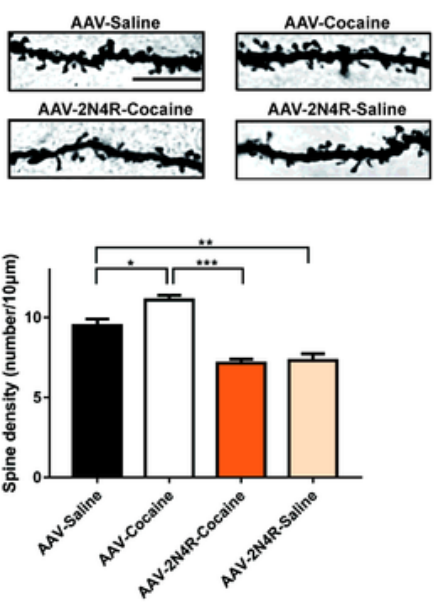

E

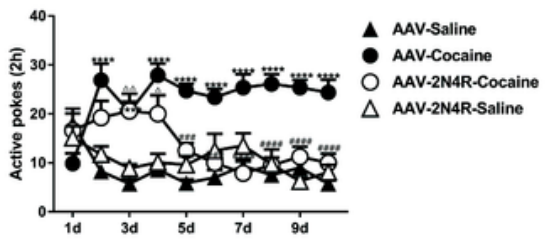

$\mathbf{F}$

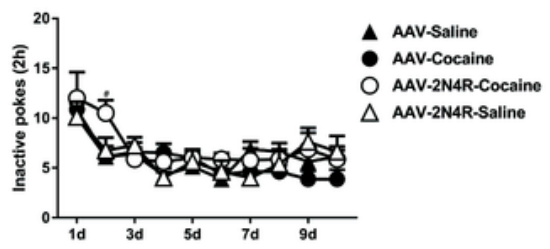

G

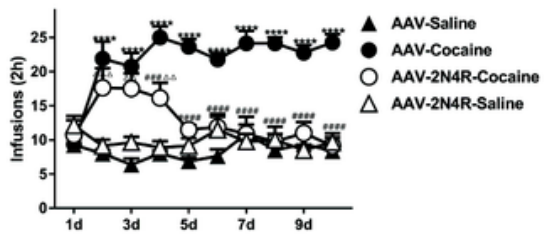

J

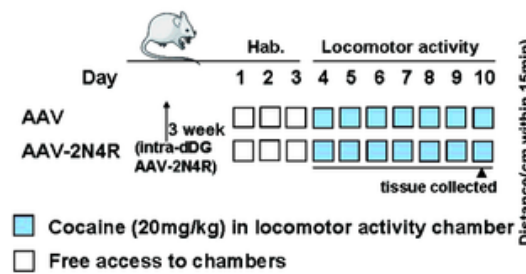

$\square$ Free access to chambers
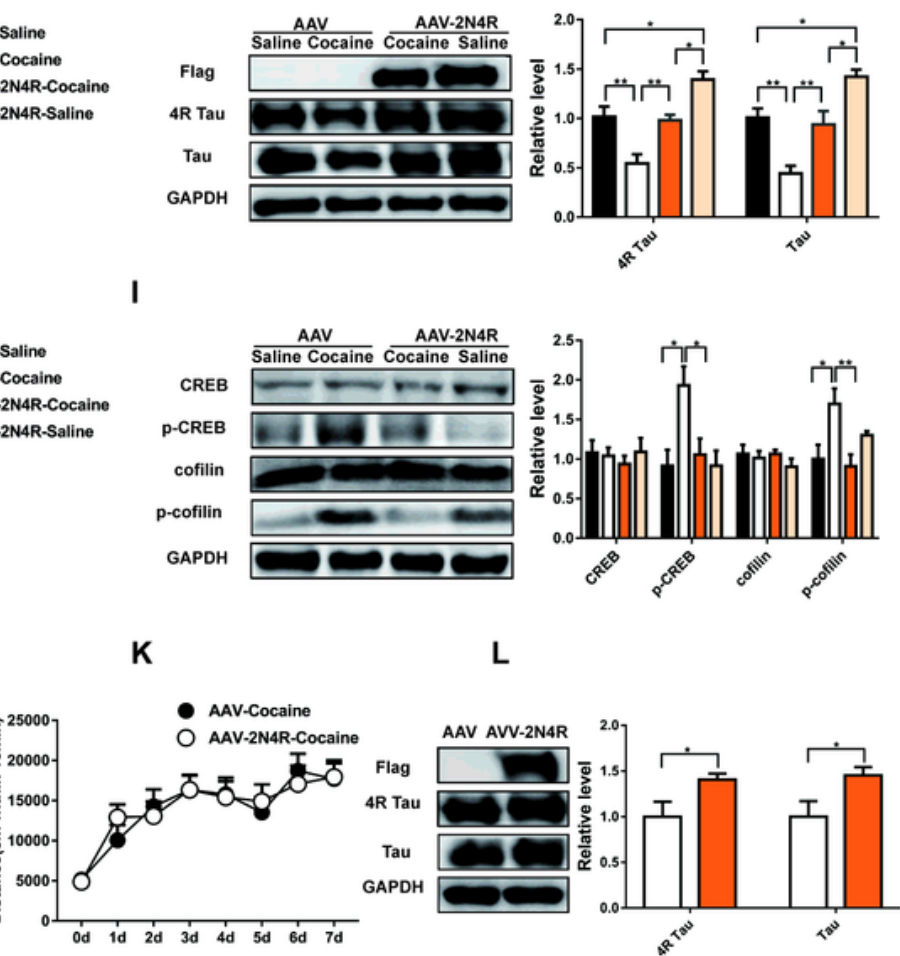

- AAV-Saline

口 AAV-Cocaine

$\square$ AAV-2N4R-Cocaine

口 AAV-2N4R-Saline
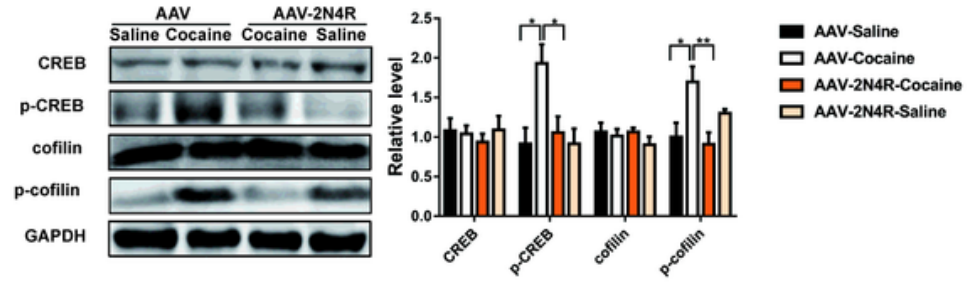

$\mathbf{L}$
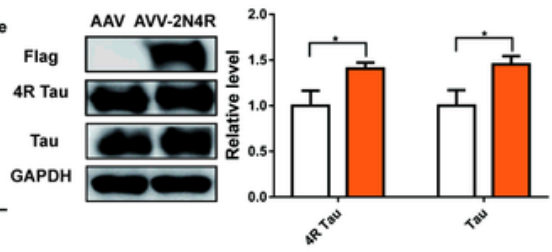

ㅁ AAV-Cocaine

口 AAV-2N4R-Cocaine

\section{Figure 4}

AAV-mediated 4R Tau overexpression modulates the expression of memory formation-related proteins and granule neurons morphology during the cocaine memory formation. a, b Expression of Flag, 4R Tau, Tau, CREB, phosphorylated CREB, cofilin and phosphorylated cofilin after cocaine CPP paradigm $(n=5$ per group). $c$ Golgi staining showed the dendritic spines density of granule neurons in the dDG ( $n=3$ per group). $d$, j Schematics of AAV-mediated 4R Tau overexpression in cocaine self-administration and 
hyperlocomotor activity tests. (e-g) Number of active pokes, inactive pokes and infusions after AAVmediated $4 R$ Tau overexpression in the dDG in cocaine self-administration $(n=8$ per group; $* \star \star * p<0.001$, AAV-cocaine group vs AAV-saline group; \#\#\#p<0.001 and \#\#\#\#p<0.0001, AAV-2N4R-cocaine group vs AAV-cocaine group; $\Delta p<0.05$ and $\Delta \Delta p<0.01, A A V-2 N 4 R$-cocaine group vs AAV-2N4R-saline group). $h$, $i$ Immunoblotting of Flag, 4R Tau, Tau as well as CREB, phosphorylated CREB, cofilin and phosphorylated cofilin in the dDG with cocaine self-administration paradigm ( $n=5$ per group). $k$ Total distance within 15 min after daily cocaine administration ( $n=8$ per group). I Flag, 4R Tau and Tau expression in the dDG after hyperlocomotion paradigm ( $n=8$ per group). Data are the means $\pm S E M,{ }^{*} p<0.05,{ }^{\star *} p<0.01,{ }^{\star \star \star} p<0.001$. 
A

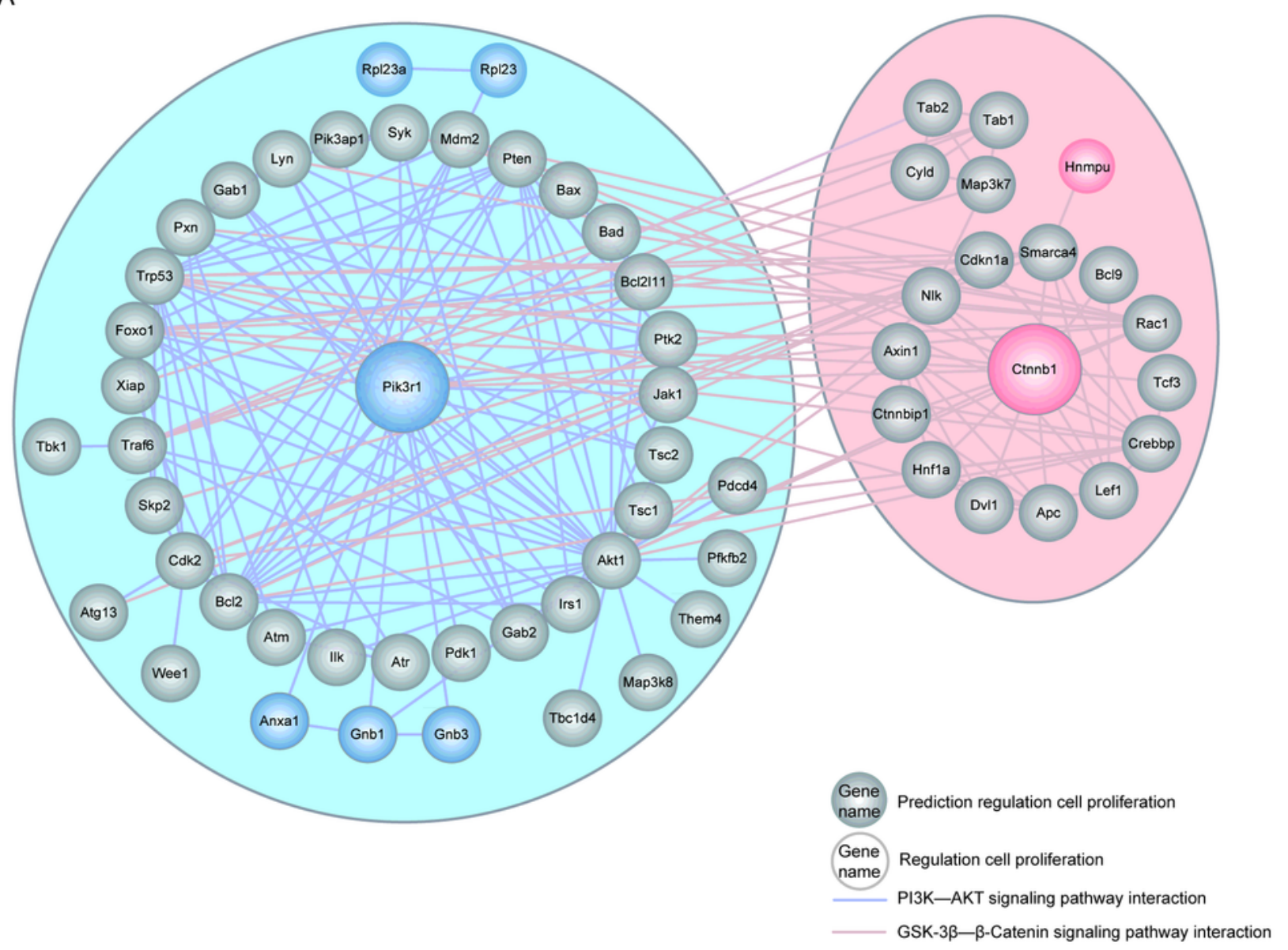

B
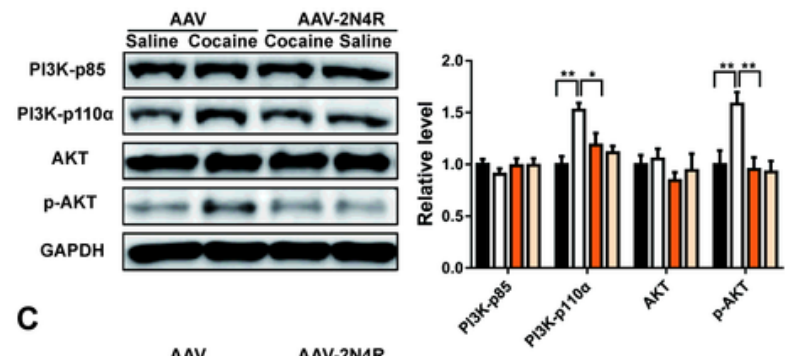

D

C

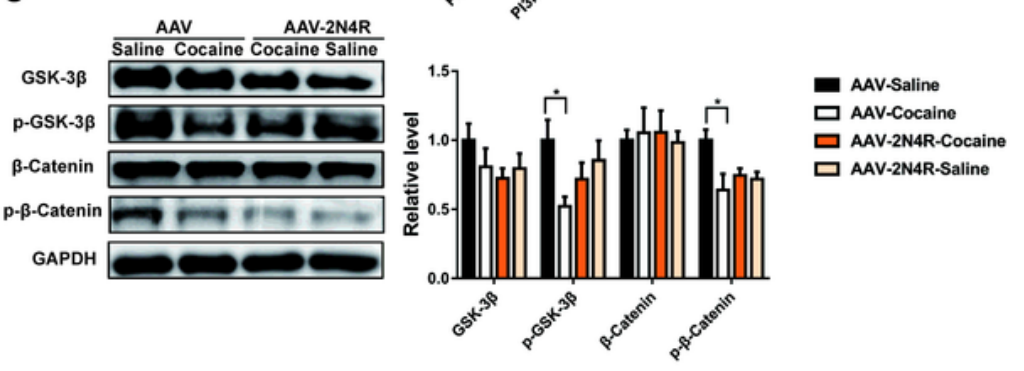

\section{Figure 5}

Analysis of Tau-interacting proteins involved in regulating cell proliferation, and AAV-mediated 4R Tau overexpression weakens PI3K-AKT singling in cocaine CPP test. a The genes involved in regulating cell proliferation are mainly distributed the PI3K-AKT (light blue) and GSK-3 $\beta-\beta$-catenin (light red) signaling pathway. Prediction gene refer to the typical genes involved in PI3K-AKT and GSK-3 $\beta-\beta$-catenin signaling, which were identified by bioinformatics analysis. b, c Western blot analysis for the expression of PI3K- 
p85, PI3K-p110a, AKT, GSK-3 $\beta$ and $\beta$-catenin ( $n=6$ per group). $d$ Endogenous PI3K-p85 was immunoprecipitated with antibodies against PI3K-p85 from tissue lysates. Rabbit IgG was used as control ( $n=3$ per group). Data are the means \pm SEM, ${ }^{*} p<0.05$ and ${ }^{* *} p<0.01$.

A

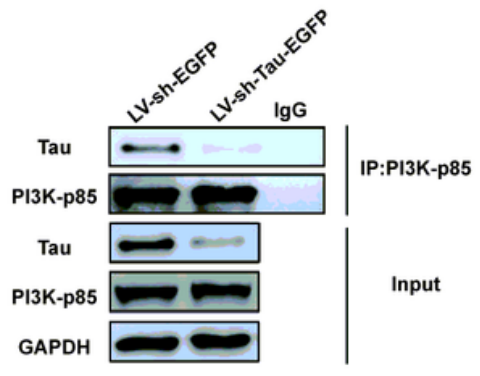

C

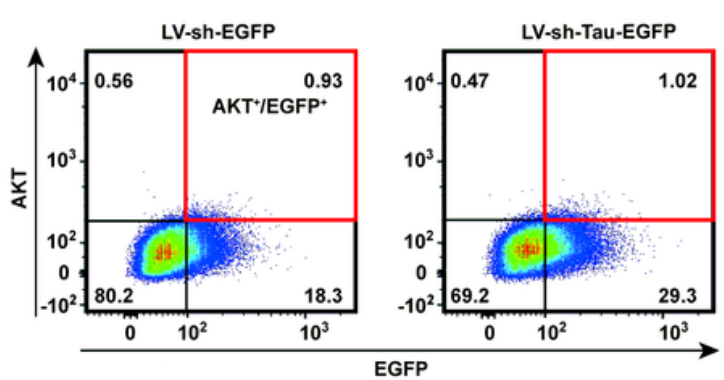

D
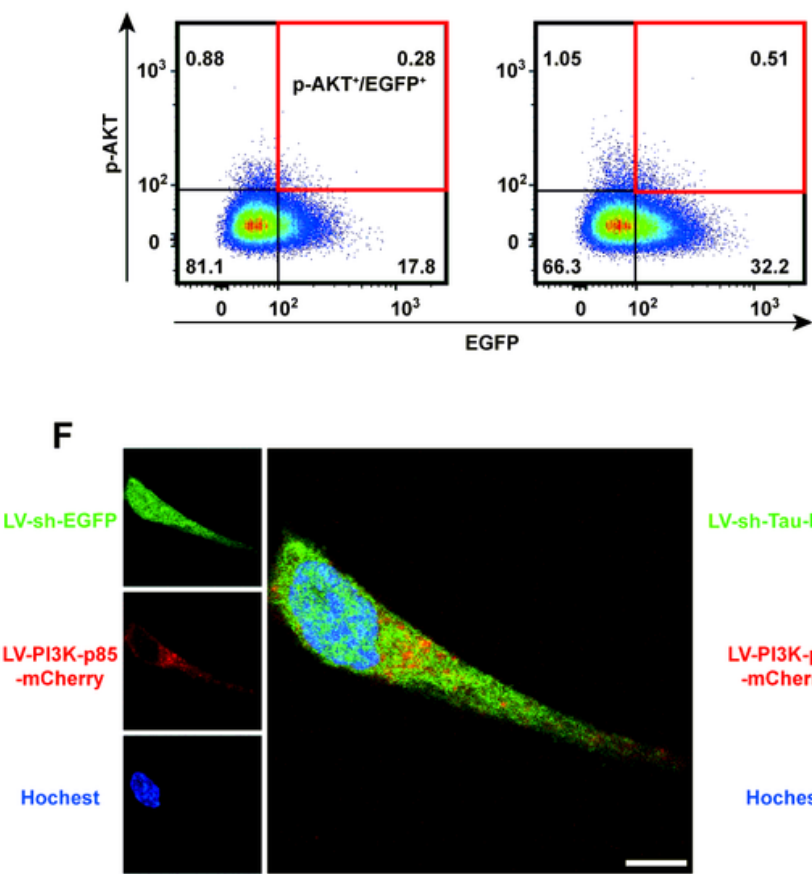

B
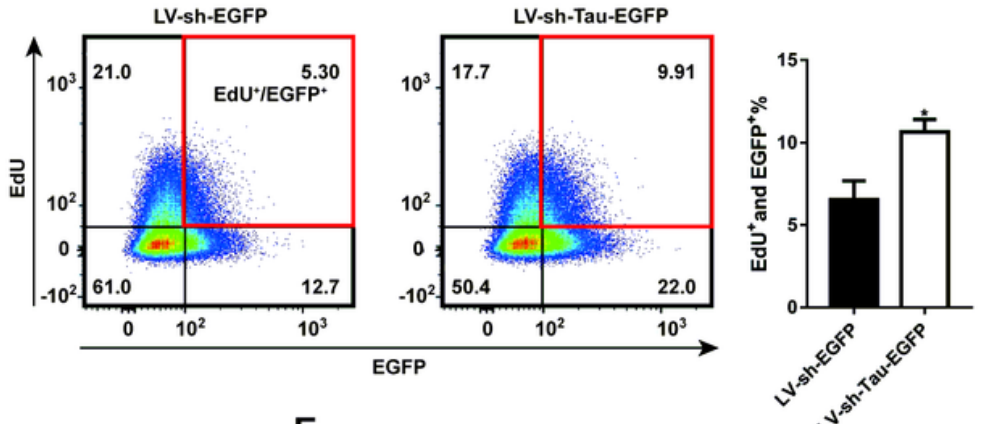

E
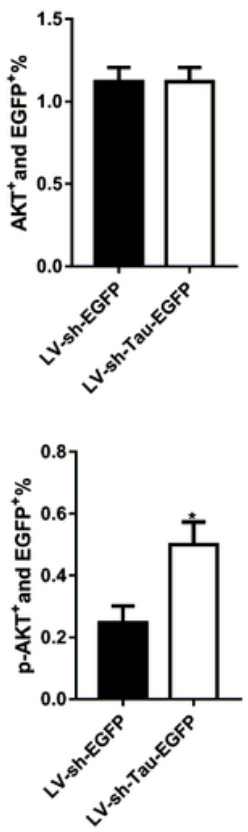

LV-sh-EGFP
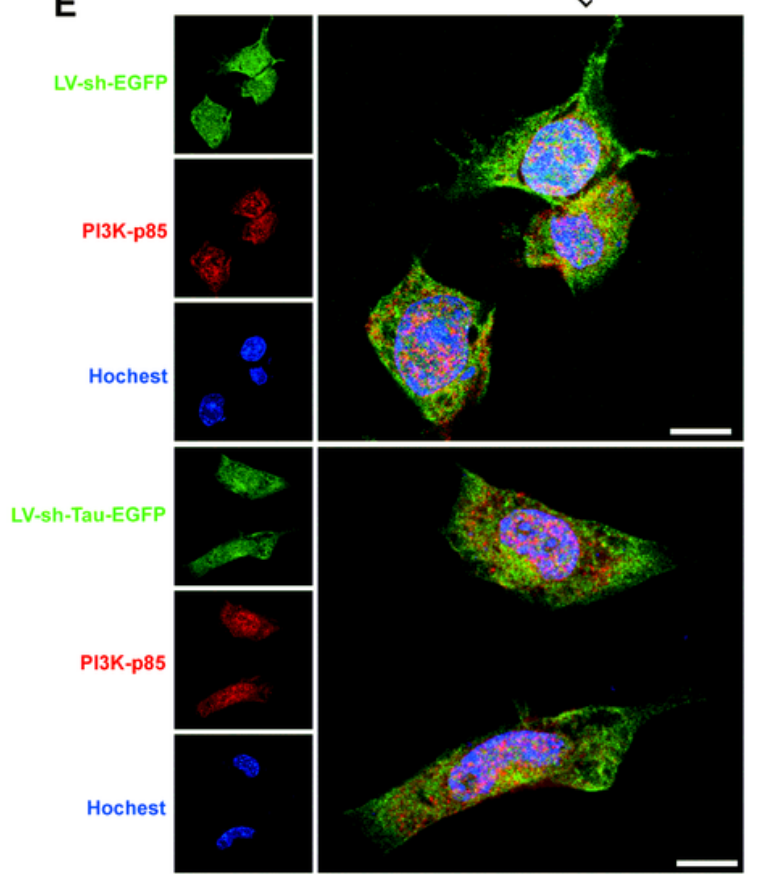

G
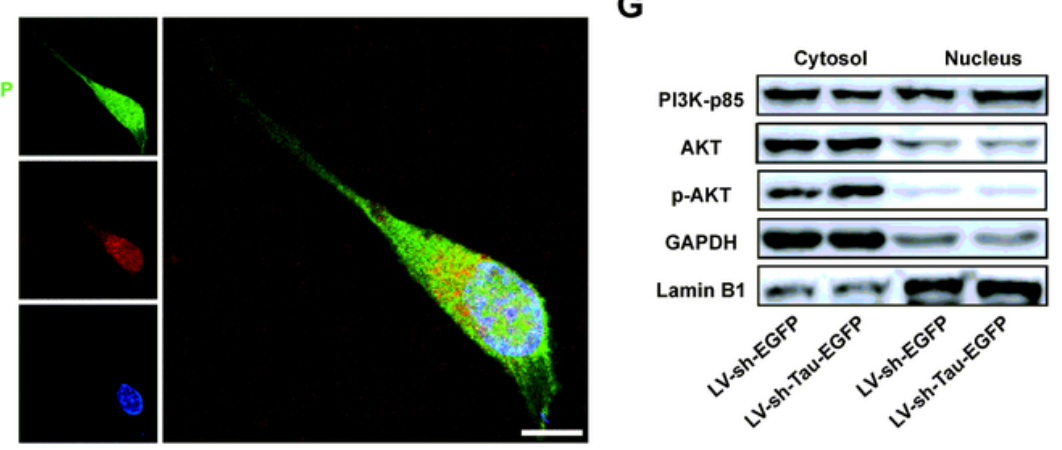

\section{Figure 6}

Silencing of Tau attenuates the interaction between Tau and PI3K-p85 and promotes PI3K-p85 nuclear translocation. a N2a cells were transfected with LV-sh-EGFP and LV-sh-Tau-EGFP, and immunoprecipitates were analyzed by immunoblotting with antibody against Tau and PI3K-p85 ( $n=3$ per group). (b-d) After exposure of external stimuli, the rates of the cell population positive for EdU, AKT and phosphorylated AKT was analyzed by flow cytometry ( $n=3$ per group). e N2a cells were transfected with 
LV-sh-EGFP and LV-sh-Tau-EGFP, and the location of endogenous PI3K-p85 was analyzed by immunofluorescence ( $n=3$ per group). $f$ Cells were transfected with LV-sh-EGFP and LV-sh-Tau-EGFP, and then transfected with exogenous LV-PI3K-p85-mCherry ( $n=3$ per group). $g$ Proteins in the cytosolic and nuclear fraction of N2a cells were analyzed by immunoblotting ( $n=3$ per group). Scale bar for $10 \mu \mathrm{m}$. Data are the means $\pm S E M,{ }^{*} p<0.05$. EGFP visualizes directly the silencing of Tau expression of N2a cells; mCherry visualizes directly the exogenous PI3K-p85 expression.

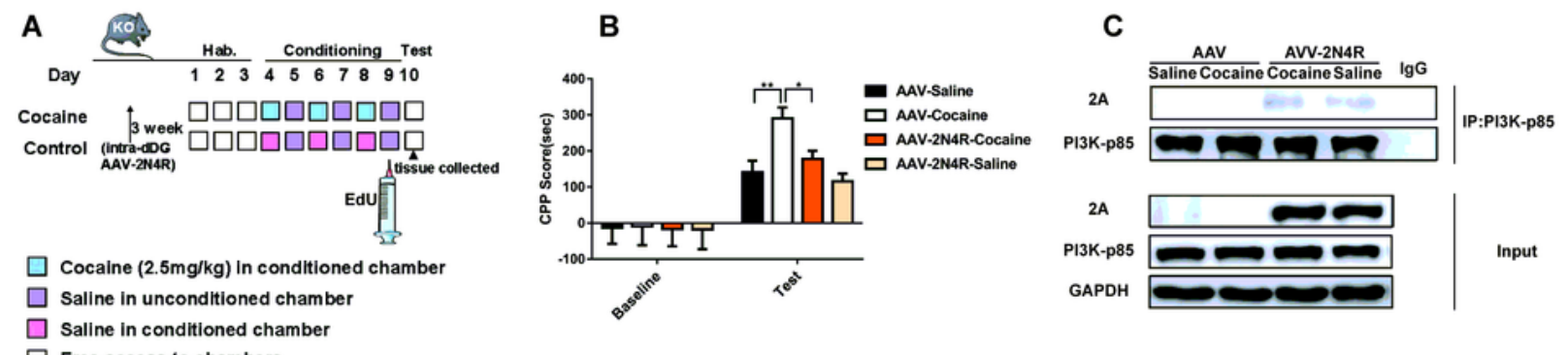

D

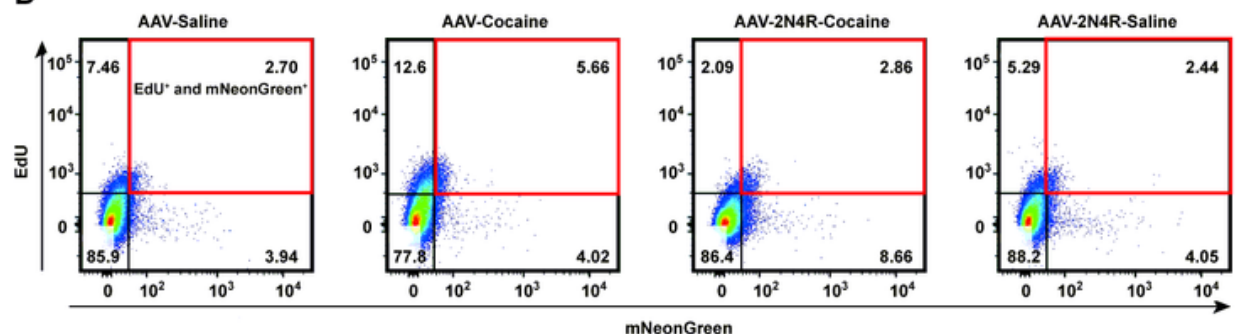

E
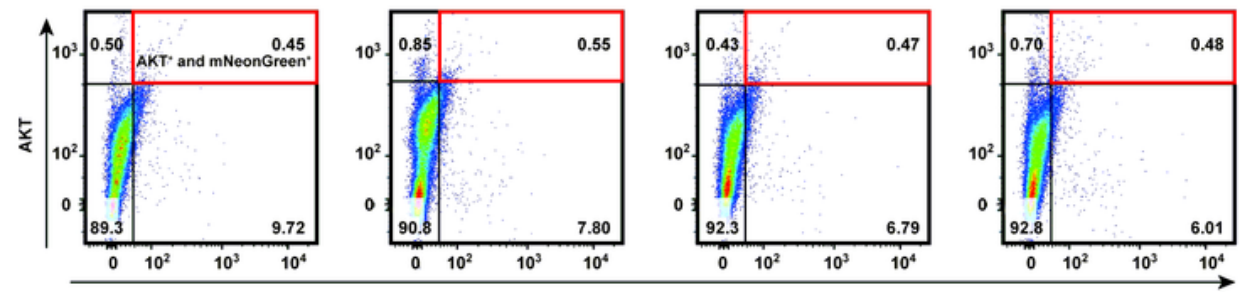

mNeonGreen

F
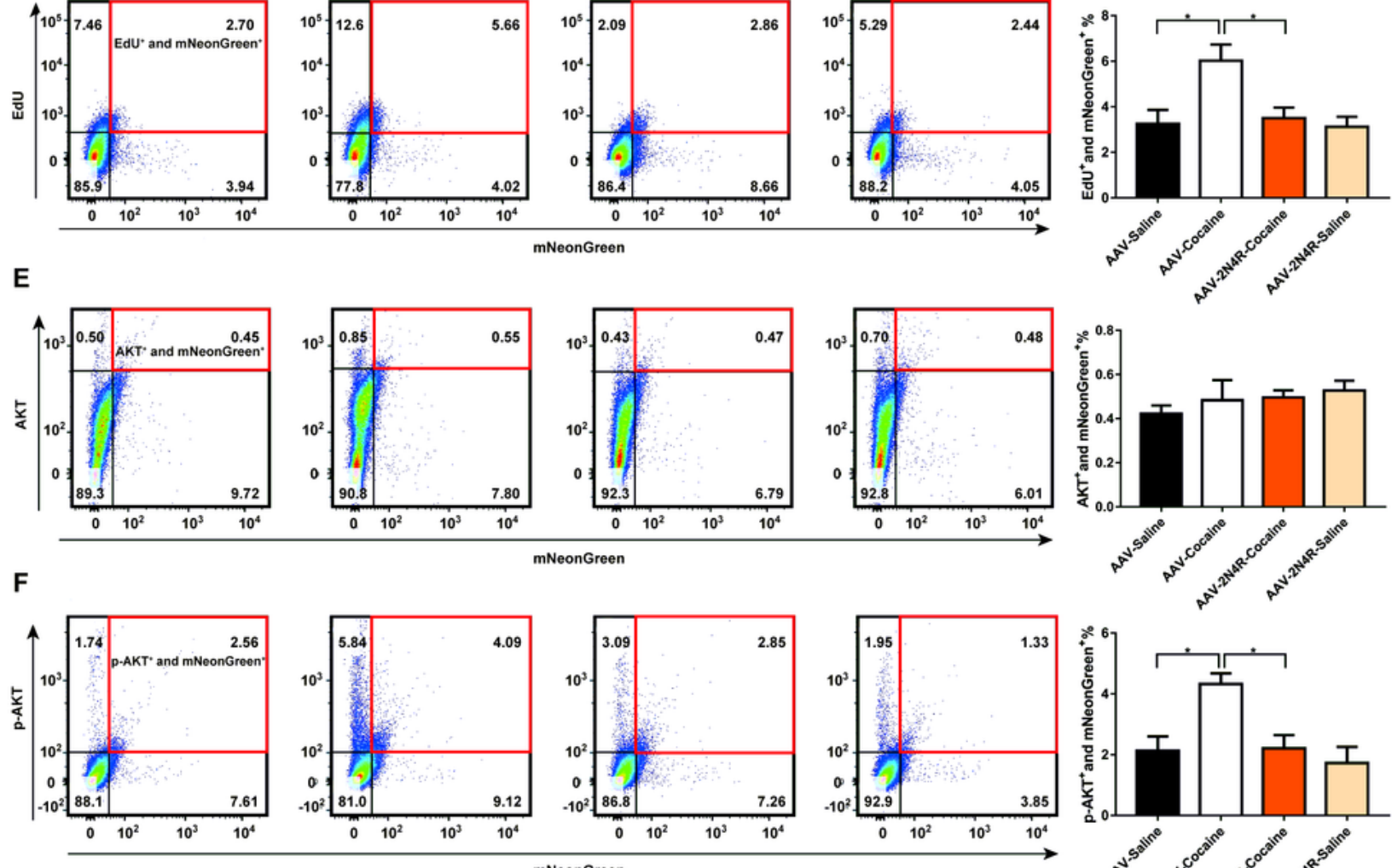

mNeonGreen

G

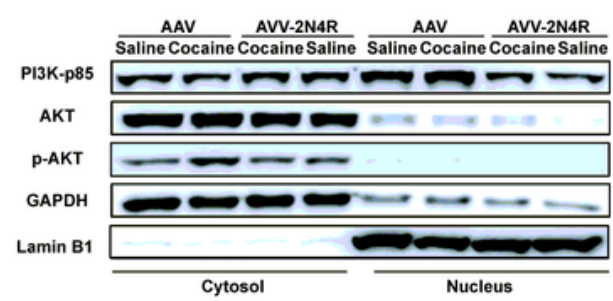

H

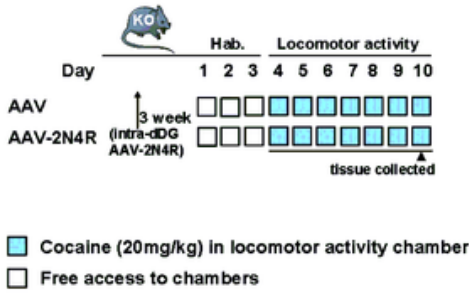

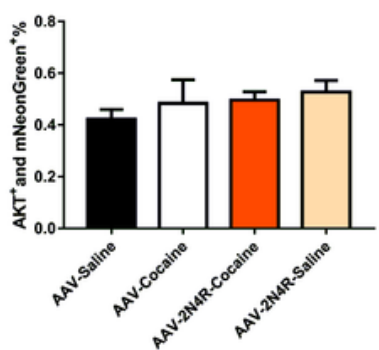

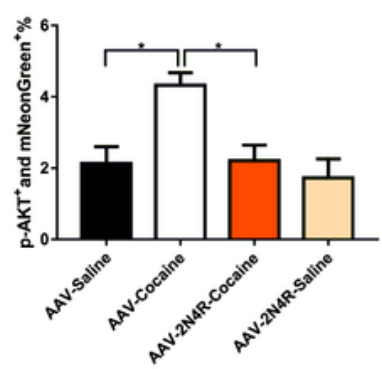

$I_{2 A}$
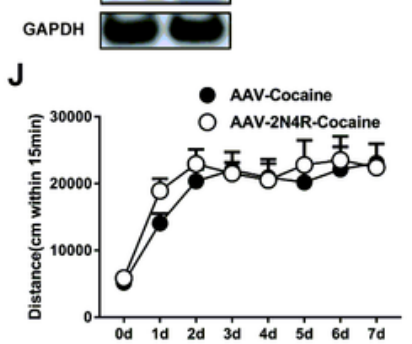
Tau regulates AHN by interacting with PI3K-p85 in the dDG during cocaine memory formation. a Schematic diagram of re-expression 2N4R Tau and EdU injection in Tau-KO mice with cocaine CPP conditioning. b Re-expression 2N4R Tau in the dDG significantly attenuated cocaine CPP ( $n=9$ per group). c Endogenous PI3K-p85 was immunoprecipitated with antibodies against PI3K-p85 from tissue lysates ( $n=3$ per group). Rabbit immunoglobulin $\mathrm{G}(\mathrm{IgG})$ was used as control. $(\mathrm{d}-\mathrm{f})$ The rates of the cell populations positive for EdU, AKT and phosphorylated AKT in the hippocampus were analyzed by flow cytometry ( $\mathrm{n}=3$ per group). $\mathrm{g}$ Re-expression of 2N4R Tau disrupts the nuclear translocation of PI3K-p85 in the $d D G$ with cocaine CPP paradigm ( $n=3$ per group). $h$ Schematics of re-expression of 2 N4R Tau in Tau$\mathrm{KO}$ mice in cocaine-induced hyperlocomotion. i Immunoblotting of $2 \mathrm{~A}$ expression ( $n=6$ per group). $\mathrm{j}$ Total distance within $15 \mathrm{~min}$ after daily cocaine administration ( $\mathrm{n}=7$ per group). Data are the means \pm SEM, ${ }^{*} \mathrm{p}<0.05$ and ${ }^{* *} \mathrm{p}<0.01$. mNeonGreen visualizes directly the expression of $2 \mathrm{~N} 4 \mathrm{R}$ Tau; $2 \mathrm{~A}$ refers to a $2 \mathrm{~A}$ peptide, a tag protein representing 2N4R Tau expression.

\section{Supplementary Files}

This is a list of supplementary files associated with this preprint. Click to download.

- supplement1.xls

- supplement2.tif

- supplement3.tif

- supplement4.tif

- supplement5.tif

- supplement6.tif

- supplement7.tif

- supplement8.tif 